\title{
Variable Stars in the Fornax dSph Galaxy. III. The Globular Cluster Fornax $5^{1}$
}

\author{
Claudia Greco, ${ }^{2,3}$ Gisella Clementini, ${ }^{2}$ Márcio Catelan, ${ }^{4,5,6}$ Enrico V. Held, ${ }^{7}$ Ennio Poretti, ${ }^{8}$ \\ Marco Gullieuszik, ${ }^{7}$ Marcella Maio, ${ }^{2}$ Armin Rest, ${ }^{9}$ Nathan De Lee, ${ }^{10}$ Horace A. Smith, ${ }^{11}$ \\ Barton J. Pritzl ${ }^{12}$
}

\begin{abstract}
We present a new study of the variable star population in globular cluster 5 of the Fornax dwarf spheroidal galaxy, based on $B$ and $V$ time series photometry obtained with the MagIC camera of the $6.5 \mathrm{~m}$ Magellan Clay telescope and
\end{abstract}

\footnotetext{
${ }^{1}$ This paper includes data gathered with the $6.5 \mathrm{~m}$ Magellan telescope located at Las Campanas Observatory, Chile, and WFPC2 at HST archival data.

${ }^{2}$ INAF, Osservatorio Astronomico di Bologna, via Ranzani 1, I-40127 Bologna, Italy; gisella.clementini@oabo.inaf.it

${ }^{3}$ Current address: Observatoire de Geneve, 51, ch. Des Maillettes, CH-1290 Sauverny, Switzerland; claudia.greco@unige.ch

${ }^{4}$ Departamento de Astronomía y Astrofísica, Pontificia Universidad Católica de Chile, Av. Vicuña Mackenna 4860, 782-0436 Macul, Santiago, Chile; mcatelan@astro.puc.cl

${ }^{5}$ John Simon Guggenheim Memorial Foundation Fellow

${ }^{6}$ On sabbatical leave at Michigan State University, Department of Physics and Astronomy, 3215 Biomedical and Physical Sciences Bldg., East Lansing, MI 48824

${ }^{7}$ INAF, Osservatorio Astronomico di Padova, vicolo dell'Osservatorio 5, I-35122 Padova, Italy; enrico.held@oapd.inaf.it, marco.gullieuszik@oapd.inaf.it

${ }^{8}$ INAF, Osservatorio Astronomico di Brera, via E. Bianchi 46, 23807 Merate, Italy; ennio.poretti@brera.inaf.it

${ }^{9}$ Physics Department, Harvard University, 17 Oxford Street, Cambridge, MA 02138; arest@physics.harvard.edu

${ }^{10}$ Department of Astronomy, University of Florida, 211 Bryant Space Center, Gainesville, FL 31611-2055; ndelee@astro.ufl.edu

${ }^{11}$ Department of Physics and Astronomy, Michigan State University, East Lansing, MI 48824-2320; smith@pa.msu.edu

${ }^{12}$ University of Wisconsin Oshkosh, Physics and Astronomy Department, Oshkosh, WI 54901; pritzlb@uwosh.edu
} 
complementary HST archive data. Light curves and accurate periodicities were obtained for 30 RR Lyrae stars and 1 SX Phoenicis variable. The RR Lyrae sample includes 15 fundamental-mode pulsators, 13 first-overtone pulsators, 1 candidate double-mode pulsator and one RR Lyrae star with uncertain type classification.

The average and minimum periods of the ab-type RR Lyrae stars, $\langle P a b\rangle=$ 0.590 days, $P_{a b, m i n}=0.53297$ days and the position in the horizontal branch typemetallicity plane, indicate that the cluster has Oosterhoff-intermediate properties, basically confirming previous indications by Mackey \& Gilmore (2003b), although with some differences both in the period and type classification of individual variables.

The average apparent magnitude of the Fornax $5 \mathrm{RR}$ Lyrae stars is $\langle V(\mathrm{RR})\rangle=21.35 \pm 0.02 \mathrm{mag}(\sigma=0.07 \mathrm{mag}$, average on 14 stars more likely belonging to the cluster, and having well sampled light curves). This value leads to a true distance modulus of $\mu_{0}=20.76 \pm 0.07\left(d=141.9_{-4.5}^{+4.6} \mathrm{kpc}\right)$ if we adopt for the cluster the metal abundance by Buonanno et al. $(1998 ;[\mathrm{Fe} / \mathrm{H}]=-2.20 \pm 0.20)$, or $\mu_{0}=20.66 \pm 0.07\left(d=135.5_{-4.3}^{+4.4} \mathrm{kpc}\right)$, if we adopt Strader et al.'s (2003) metal abundance $([\mathrm{Fe} / \mathrm{H}]=-1.73 \pm 0.13)$.

Subject headings: galaxies: dwarf — galaxies: individual (Fornax) - globular clusters: individual (Fornax 5) — stars: horizontal branch — stars: variables: other - techniques: photometry

\section{Introduction}

This is the third in a series of papers devoted to the study of the variable star population of the Fornax dwarf spheroidal galaxy (dSph) based on a photometric survey that reaches $V \sim 25-26$ mag and covers about $1 \mathrm{deg}^{2}$ of the galaxy field and all the 5 globular clusters belonging to Fornax (see Clementini et al. 2006, for a description of the whole project). In Greco et al. (2007, Paper I) we have presented the first study of the variable star population of Fornax 4 (hereafter For 4), the cluster located towards the centre of Fornax, which Hardy (2002) and Strader et al. (2003) suggest might be the galaxy nucleus. In Poretti et al. (2008, Paper II) we reported results on the identification of 85 SX Phoenicis stars in a $33^{\prime} \times 34^{\prime}$ North portion of the Fornax dSph field. In the present paper we present results for variable stars in the globular cluster Fornax 5 (hereafter For 5 ), that, along with Fornax 1 (hereafter, For 1), is the most external of the Fornax clusters. In the following papers of the series we will report on the study of the brighter variables (RR Lyrae stars and Anomalous Cepheids) 
in the Fornax field, as well as in the globular clusters Fornax 3 (hereafter, For 3, Clementini et al. 2009, in preparation), For 1 and Fornax 2 (hereafter, For 2).

The 5 globular clusters of the Fornax dSph galaxy (Hodge 1961, 1965, 1969) form a complex system with no straightforward correlations existing between galactocentric distance, metallicity and age. They are all metal poor $(-2.0 \leq[\mathrm{Fe} / \mathrm{H}] \leq-1.5)$, have rather similar mass and are distributed at varying distances from the galaxy center marked by For 4 . For 5 is placed at the galaxy periphery, about $26^{\prime}$ North and $33.5^{\prime}$ East of For 4 . According to the surface brightness profile it seems a post-collapse cluster with core radius $r_{c}=2.08 \pm 0.17$ arcsec (Mackey \& Gilmore 2003a). The literature values for the cluster tidal radius range from $r_{t}=64 \pm 18 \operatorname{arcsec}$ (Webbink 1985), to $r_{t}=74 \pm 5 \operatorname{arcsec}$ (Demers et al. 1994), to $r_{t}=91 \operatorname{arcsec}($ Smith et al. 1996).

Buonanno et al. (1998) published a color magnitude diagram (CMD) for the cluster based on Hubble Space Telescope (HST) Wide Field Planetary Camera 2 (WFPC2; Program ID 5917) observations, from which they estimated the cluster relative age and metallicity. The CMD is rich in stars, with a horizontal branch (HB) extending to the blue across the RR Lyrae star instability strip, and suggests that For 5 is coeval with the oldest Galactic globular clusters. Buonanno et al. (1998) also find that For 5 is metal poor, with $[\mathrm{Fe} / \mathrm{H}]=-2.20 \pm 0.20$ dex, on the Zinn \& West (1984) metallicity scale. This metallicity is in good agreement with previous estimates, from both photometric (Buonanno et al. 1985) and spectroscopic studies (Dubath, Meylan \& Mayor 1992).

Strader et al. (2003) published new age and metal abundance estimates for the cluster based on low-resolution, integrated Keck spectra. They derive a larger metallicity of $[\mathrm{Fe} / \mathrm{H}]=-1.73 \pm 0.13$ (on the Zinn \& West 1984 metallicity scale). They also suggest that For 5 is a few billion years younger than the other clusters in Fornax.

Buonanno et al. (1998) found evidence for 36 possible variables in For 5, on the basis of the star frame-to-frame variations in their HST data, but did not provide identification or measurement of any variables. More recently, Mackey \& Gilmore (2003b; hereafter MG03) presented a more quantitative study of the RR Lyrae stars in For 5, based on the same HST dataset used by Buonanno et al. (1998) to build the cluster CMD. The small number (14-16 images) and short time interval (8-9 consecutive hours) spanned by the HST archive data were inadequate to allow MG03 a direct determination of the periods, and they fitted template light curves of RR Lyrae stars to the data to get an estimate of periodicity and amplitude of the light variation. Based on their period estimates MG03 concluded that the RR Lyrae stars in For 5 have mean pulsation period intermediate between the two Oosterhoff types (Oosterhoff 1939) observed within the MW GCs. 
In this paper we present results from a new study of the variable star population in For 5, that resulted in the identification and in the definition of accurate pulsation parameters for 30 RR Lyrae stars and one SX Phoenicis (SX Phe) variable. Seven of the RR Lyrae stars and the SX Phe variable are new discoveries, that do not have a counterpart in the MG03 study. The periods, amplitudes, and period-amplitude distributions of the RR Lyrae stars allowed us to firmly establish the Oosterhoff type (Oo-type) of the cluster. Their average luminosity was used to measure the cluster distance.

Observations and data reductions are presented in Sect. 2. The variable star identification is described in Sect. 3. The period search technique, the pulsation properties of the For 5 variable stars, and their membership in the parent cluster are discussed in Sect. 4. In this section we also compare our results with the MG03 study. In Sect. 5 we discuss the Oosterhoff classification of For 5 and compare it to For 4, for which in Paper I we found an Oosterhoff-Intermediate behavior. In Sect. 6 we derive the cluster distance from the average luminosity of the RR Lyrae stars. Final results are summarized in Sect. 7.

\section{Observations and data reductions}

$B, V$ time series photometry of For 5 was obtained over two consecutive nights in November 2003 with the MagIC camera of the Magellan/Clay 6.5-m telescope, equipped with a $2048 \times 2048$ SITe CCD having resolution of 0.069 arcsec pixel ${ }^{-1}$. Observations of For 4 were also obtained during the same nights (see Paper I). We covered a total field of view $(\mathrm{FOV})$ of $2.4^{\prime} \times 2.4^{\prime}$, centered on the cluster at R.A. $=2^{h} 42^{m} 21.07^{s}, \mathrm{DEC}=-34^{\circ} 06^{\prime} 07.5^{\prime \prime}$ (J2000). According to the tidal radii provided by Webbink (1985) and Demers et al. (1994) for For 5, our FOV should cover the cluster almost in its entirety. Nights were photometric with seeing conditions varying from $0.45^{\prime \prime}$ to $0.65^{\prime \prime}$. We acquired $52 \mathrm{~V}$ and $20 \mathrm{~B}$ images of For 5. Average exposure times were of 500 seconds for the $V$ frames and $700 \mathrm{~s}$ for the $B$ ones. Observations of the standard fields $\mathrm{T}$ Phe and $\mathrm{Ru} 149$ (Landolt 1992) were obtained to calibrate the data to the standard Johnson-Cousins photometric system.

The Magellan frames were bias-subtracted and flatfield-corrected using the MagIC tool in IRAF1. PSF photometry was performed with DAOPHOT-ALLSTAR and time series photometry for all the stars was produced with ALLFRAME (Stetson 1994). The internal photometric precision at the HB level is of 0.01 mag. The photometric calibration of the

\footnotetext{
${ }^{1}$ IRAF is distributed by the National Optical Astronomical Observatories, which are operated by the Association of Universities for Research in Astronomy, Inc., under cooperative agreement with the National Science Foundation
} 
For 5 data (as well as for For 4, Paper I) was derived using the standard stars observed at the Magellan telescope on the night of Nov. 13, 2003 (UT). A set of linear calibration relations was derived according to the procedure described in Paper I, to which the interested reader is referred to for details. The zero point uncertainties of the calibration relations are estimated of the order $0.05 \mathrm{mag}$ in $B$ and $V$, and $0.03 \mathrm{mag}$ in $(B-V)$.

The Magellan time series observations of For 5 correspond to a total observing time of $26000 \mathrm{sec}$ in $V$ and $14000 \mathrm{sec}$ in $B$ and produced a CMD reaching $V \sim 26$ mag. This CMD is shown in Fig. 1. No reliable photometry could be obtained for stars inside a radius of about $8^{\prime \prime}$ from the cluster center due to crowding. The CMD in Fig. 1 was obtained cutting off this central region.

In order to improve the light curve sampling and the period definition, the Magellan data were complemented by the HST archival data of HST Program 5917, the same dataset used in the Buonanno et al. (1998) and in the MG03 studies. The HST archival dataset comprises 14 F555W and 16 F814W images obtained over about 8-9 consecutive hours, in 1996, June, with the planetary camera (PC; resolution 0.0455 arcsec pixel ${ }^{-1}$, FOV $36^{\prime} \times 36^{\prime}$ ) and the 3 wide field (WF) cameras (resolution $0.0996 \operatorname{arcsec}$ pixel ${ }^{-1}$, FOV 80' $\times$ 80') of the WFPC2. Exposure times varied from 160 to $600 \mathrm{sec}$ for the F555W filter, and from 20 to 900 sec for the F814W filter. The reduction of the HST archival data was performed with DAOPHOTALLSTAR-ALLFRAME exactly in the same way as for the Magellan dataset. The F555W

WFPC2 instrumental magnitudes were linked to the Johnson-Cousins magnitudes of the Magellan dataset following the procedure described at the beginning of Section 4. Logs of the observations and details of the instrumental set-ups are provided in Table 1 for the Magellan and HST data, separately.

\section{Variable star identification}

Variable stars were identified by applying the Image Subtraction Technique of ISIS2.1 (Alard 2000) to the Magellan $V$ and $B$ dataset separately. Images in each photometric band were first aligned and re-mapped onto the same grid. Then $V$ and $B$ reference images were constructed by stacking the frames obtained in best seeing conditions, and each individual frame of the time-series was subtracted from the corresponding reference image, after convolution with a suitable kernel to match seeing variations and geometrical distortions on the individual images. The output of ISIS2.1 is a median image ("var.fits") of all difference frames, in which non-variable objects disappear and candidate variable stars stand out as bright/dark peaks. The last step of ISIS2.1 is to perform profile fitting photometry of the variable sources in the subtracted frames to build differential flux light curves for each can- 
didate variable star identified in the var.fits frame (see Alard 2000, for details). To exclude false detections, the $B$ and $V$ catalogues of sources showing light variations according to ISIS were cross-identified in order to select objects which consistently varied in both photometric bands. About 300 candidate variables were identified in the field of For 5, including the cluster central region. The final selection of bona-fide variables was made through visual inspection of the light curves in differential flux. A very large fraction of the candidate variables turned out to be spurious detections due to CCD bad columns, hot pixels, satellite trails, and saturated objects. The spurious origin of their variability was revealed by the almost identical light curves and from the close positions on the frames of the contamined objects. The ISIS catalog was cross-identified against the DAOPHOT/ALLFRAME photometric catalog obtaining light curves in magnitude scale from the ALLFRAME time series

photometry and confirming the variability for 21 stars. A further 10 variable stars were recovered with ISIS in the central region of For 5, where we lack reliable PSF photometry of the Magellan data. These stars have only differential flux light curves, in the Magellan dataset. By matching the coordinates the confirmed variables identified on the Magellan images were cross-identified against the photometry of the HST archival data. Twenty-six out of the 31 variable stars found in For 5 have a counterpart in the HST photometry. Of the missing five ones, four (namely stars V22, V26, V29 and V30) are outside the FOV covered by the HST observations, and one star (V5) lacks a secure counteridentification in the HST dataset since it lies in a very crowded region close to For 5 center (see also Section 4.3).

\section{Period Search and Properties of the Variable Stars}

\subsection{Analysis of the light curves}

The time series of the candidate variable stars were analyzed using GrATiS (Graphical Analyzer of Time Series), a custom software developed at the Bologna Observatory by P. Montegriffo (see Di Fabrizio 1999, Clementini et al. 2000) which uses both the Lomb periodogram (Lomb 1976, Scargle 1982) and the best fit of the data with a truncated Fourier series (Barning 1963). Both the Magellan and the HST datasets were used, when available, to determine the pulsation characteristics of the variable stars from the study of the light curves. In order to combine the HST F555W and the Magellan $v$ photometries we used a number of stable reference stars common to both datasets and having colors close to those of the candidate variables, and built instrumental differential light curves. These were then tied to the Magellan instrumental photometric system (by adding up the instrumental magnitude of the comparison stars in the Magellan photometry) and finally calibrated to the standard system using the calibration procedure discussed in Sect. 2. The time series $B, V$ 
photometry of the confirmed variable stars with light curves in magnitude scale is provided in Table 2. The full version of this table is published in the electronic edition of the Journal. Identification and coordinates of the confirmed variable stars are presented in Table 3.

We were able to derive reliable periods for the vast majority of the confirmed variable stars detected in For 5. However, the accuracy of our period determinations varies significantly (in the range from 2 to 6 digits) depending on the spectral window of the available data, whether only Magellan or Magellan + HST (see Table 3).

The Magellan observations of For 5 consist of 3.5 and 7.6 hours of consecutive exposures taken along the two nights of 2003, November 13 and 14 (UT), respectively from $\mathrm{HJD}=2452956.538$ to $\mathrm{HJD}=2452956.684$, and from $\mathrm{HJD}=2452957.518$ to $\mathrm{HJD}=2452957.836$, while the HST data were acquired along about 7.8 consecutive hours in 1996, June 4-5 (UT) (from HJD=2450238.721 to HJD=2450239.048). Magellan as well as HST photometry is available for 24 of the 31 variables we found in For 5 . They include 9 of the variables having light curves only in differential flux in the Magellan dataset. By comparing the Magellan differential flux light curves and the HST light curves in magnitude scale we were able to improve the period determination for these variables, which, however, remains more uncertain for stars with periods longer than 0.5 days. Moreover, using the HST photometry we managed to obtain a rough estimate of their $\langle V\rangle$ magnitudes that confirmed they are HB variables, we estimated the $V$ amplitudes of 4 of them, and could set lower limits to the amplitude of other 4 variables.

The definition of the period of the For 5 variables is driven by the observations taken on the two consecutive nights at the Magellan telescope. The For 5 field was surveyed for 0.46 days on these nights, allowing us to pin down periods shorter than this value in a very reliable way, whilst longer periods are more uncertain. The HST data improved the phase coverage in several cases, also refining the values of the Magellan periods. The spectral window of the For 5 data shows several peaks at multiple integers of $\pm 0.00037 \mathrm{~d}^{-1}$ around the central peak, owing to the large gap between the HST and the Magellan observations. Their power is higher than $98 \%$ as far as $\pm 0.05 \mathrm{~d}^{-1}$ from the central peak. Up to six-digits periods were necessary to fold the light curves of some of the variables listed in Table 3, but the alias structure introduces larger uncertainties. For instance, the data of V13 can be fit in equivalent manner by periods of $0.399790,0.406060$ or 0.411842 days and we cannot select the true period in an unambiguous way. However, given the small difference between alternative periods the scientific discussion is not significantly affected in this case. Similarly, among the long-period RR Lyrae stars, the light curve of V17 is fitted by $P=0.68$ days but we cannot completely rule out a longer period for this star, while the data of V20 are fitted equally well by periods of 0.74740 and 0.769393 days. In the latter case, the period 
difference is not negligible and we took the period uncertainties into account in the scientific discussion. The uncertain periods have been flagged in Table 3. In a few cases where a shorter period corresponding to first-overtone pulsation, and a longer period corresponding to fundamental mode pulsation, were both likely, we used the shape, amplitude and timerise to the maximum of the light curve (if available) to achieve the final classification, which generally turned out to be as a fundamental mode pulsator.

The cluster variable stars include $30 \mathrm{RR}$ Lyrae stars and one SX Phe variable. The RR Lyrae sample comprises $15 a b$-, 13 c-type, 1 candidate double-mode pulsator ( $d$-type, star V18), and a further RR Lyrae star, variable V15, whose period definition and type classification are uncertain. The properties (pulsation period, type, time of maximum light, number of phase points in the $V$ and $B$ bands, intensity-averaged $\langle\mathrm{V}\rangle$ and $\langle\mathrm{B}\rangle$ magnitudes, and amplitudes of the light variation, $A_{V}$ and $A_{B}$ ) of the variable stars are summarized in Table 3. Examples of light curves are shown in Fig. 2. The complete atlas of the RR Lyrae stars light curves is presented in Figs. 3 and 4 . The light variation of the For 5 RR Lyrae stars is generally well sampled in both the $V$ and the $B$ photometric bands, with standard deviations of the least-square fits of the light curves in the range from 0.02 to 0.05 mag, and from 0.02 to 0.06 mag in $V$ and $B$, respectively.

The scatter and, specifically, the two maxima with slightly different shape observed in the light curves of V18 on two consecutive nights suggest that the star could be a doublemode RR Lyrae variable. Indeed, we find that a solution with fundamental mode period of $P_{0}=0.49602$ days and period ratio of $P_{1} / P_{0}=0.74640$, is possible, although not as the best solution. In fact, the phase coverage is incomplete and the peak of one term is perturbed by the alias structure of the other. It is also noteworthy that there are other RRc variables in For 5 with longer periods than V18 that do not seem to be double-mode pulsators, V19 with a slightly longer period than V18 shows a little scatter and some evidence for a double maximum light, but V28, V26 and V13, with significantly longer periods, seem to show little sign of an excess light curve scatter. Additional data are needed to establish the double mode nature of V18, that, if confirmed, would bring to 7 the number of RRd stars detected so far in extragalactic globular clusters: 4 are reported in the Reticulum cluster of the Large Magellanic Cloud (Ripepi et al. 2004) and two RRd's were found in Fornax 4 by Greco et al. (2007, Paper I). The frequency of RRd stars in the Fornax dSph clusters and what the presence of RRd stars in extragalactic clusters may imply will be discussed in more detail in a following paper of this series (Clementini et al. 2009, in preparation).

V29 is the only short-period $(P<0.1$ days $)$ variable detected in our survey of For 5 . Despite the star's faintness $(V \sim 23.7)$, the peak at $f=20.20 \mathrm{~d}^{-1}$ stands out clearly in the amplitude spectrum of the $V$ data, with a signal-to-noise ratio of 7.0. Being located beyond 
45 arcsec from the cluster center, the star probably belongs to the Fornax field. The period (0.0495 days) and the $\langle B\rangle$ and $\langle V\rangle$ magnitudes (23.97 and 23.74 mag, respectively) put the star close to the $P-L$ relation of the Fornax SX Phe stars (see Fig. 7 in Paper II; V29 is similar to 2_V3796).

\subsection{Variable stars in the CMD}

The variable stars are plotted in the CMD of Fig. 1 according to their magnitudes and colors intensity-averaged over the full pulsation cycle, and with different symbols/colors for the first-overtone, fundamental, double-mode RR Lyrae stars and the SX Phe variable. Thanks to the good sampling of the light curves the RR Lyrae instability strip in For 5 is very well defined and appears to not extend much in color (see Fig. 1). The blue edge of the strip is at $\langle\mathrm{B}\rangle-\langle\mathrm{V}\rangle=0.22 \pm 0.03 \mathrm{mag}$, as derived from the average of the three bluest $c$-type RR Lyrae stars with secure membership to the cluster (see Sect. 4.4), namely, V21, V23 and V24. The instability strip red edge appears to be at $\langle\mathrm{B}\rangle-\langle\mathrm{V}\rangle \sim 0.40$ mag, with a rather sharp separation between RR Lyrae and red HB stars. The color of the blue edge of the RR Lyrae strip can be used to estimate the reddening of the cluster. By matching the blue edge of the RR Lyrae strip in M3 $\left[E(B-V)_{\mathrm{M} 3}=0.01\right.$ mag; Harris 1996] to the colors of the bluest RR Lyrae stars in For 5 we derive: $E(B-V)=0.04 \pm 0.03$ mag. This value can be compared with the value of $E(B-V)=0.022 \mathrm{mag}$ for the foreground Galactic extinction in the direction of For 5, derived from the Schlegel, Finkbeiner, \& Davis (1998) maps.

\subsection{Comparison with previous work}

MG03 have identified 40 RR Lyrae in For 5, of which 33 are on the PC, 2 on the WFPC22, 1 on the WFPC2-3, and 4 on the WFPC2-4. We have identified 30 RR Lyrae and one SX Phe star in For 5, of which 23 have a counterpart in MG03, namely 19 stars on the PC, 2 on the WFPC2-2, one star on the WFPC2-3, and one star on the WFPC2-4. The crossidentification of the For 5 variables with MG03 is provided in Table 4. The table comprises 22 variables classified as RR Lyrae stars in both studies and one star (V19) listed among MG03 candidate variables (see Table 3 of MG03) which we classify as a first-overtone RR Lyrae. Four out of the 22 RR Lyrae stars in common with MG03 are classified $a b$-type pulsators by these authors, but have very uncertain periods. We confirm the type classification for all of them, but derive longer periods by about $\Delta P \sim 0.16$ days, on average, with full range from 0.03 to 0.23 days. Further three stars classified as first-overtones by MG03 are instead fundamental-mode pulsators (V10 and V11) and a candidate double-mode pulsator (V18). 
We were not able to unambiguously establish the period and type classification of V15. MG03 classify this star as an RRc with $\mathrm{P}=0.394$ days. We find that $\mathrm{P}=0.340$ phases our data (see Fig. 4); however, other periods for the star are also possible. For the remaining 14 stars in common there is agreement in the type classification, 5 are $a b$-type and 9 are $c$-type pulsators according to both studies. Among the $a b$-type pulsators, three have periods underestimated by 0.07 days, on average, with full range from 0.01 to 0.14 days; while 2 have periods overestimated by 0.05 days in MG03. Among the $c$-type pulsators, for V31 we fully confirm MG03's period determination, four have periods underestimated by 0.02 days, on average, with full range from 0.01 to 0.05 days; while 4 have periods overestimated by 0.005 days, on average, with full range from 0.001 to 0.01 days, in MG03. The comparison between our period determinations and MG03 values for the stars in common is shown in Fig. 5, where we plot the difference between our and MG03 estimates as a function of our periodicities. Open and filled circles represent first-overtone and fundamental-mode pulsators, respectively, according to our type classification. In our sample there are 3 variables on the PC (namely, stars V1, V5, V17) and one variable star on the WFPC2-4 (V24) that have no counterpart in MG03. V1 and V5 are in the very central region of For 5. They were detected with the image subtraction technique, however, no reliable measure of magnitude could be obtained for them either in the HST or in the Magellan dataset. Star V17 has very small amplitudes. These reasons may explain why MG03 missed these three variable stars. Finally an additional 4 variables in our sample (namely, stars V22, V26, V29, and V30) are outside the field covered by the HST observations.

\subsection{Membership Probability}

We checked the contamination of the For 5 RR Lyrae stars by field variables. Given the different stellar content of the globular cluster and the surrounding Fornax field populations, and the presence of a stellar population gradient in Fornax, estimating the expected number of cluster RR Lyrae variables from the surface density profile in our small observed field is not straightforward. To illustrate the difference between the stellar populations of the cluster and the field, we have selected stars in two regions, inner and outer. Then we have used the outermost variable stars in the field to empirically estimate the expected surface density of RR Lyrae stars in the field of the Fornax galaxy.

The count excess produced by the stars in the globular cluster For 5 is very small beyond $R=45^{\prime \prime}$. This is consistent with the density profile of For 5 obtained by Mackey \& Gilmore (2003a). In our Magellan data, 2 RR Lyrae stars (one fundamental-mode, V30, and one first-overtone, V31, the bluest RR Lyrae in the field of For 5, with $\langle\mathrm{B}\rangle-\langle\mathrm{V}\rangle=0.15 \mathrm{mag}$ ) 
have been identified beyond $45^{\prime \prime}$, in an area of $1.4 \times 10^{4} \operatorname{arcsec}^{2}$. On the basis of the overall surface density profile of For 5 , for statistical purposes we assume that these variables are not associated with the cluster. This provides an upper limit to the number density of field variable stars. Then we estimate a surface density of $1.5 \times 10^{-4} \mathrm{RR}$ Lyrae per $\operatorname{arcsec}^{2}$ in the adjacent Fornax field. In the inner region, within a radius of $45^{\prime \prime}$ from the center, 0.9 field RR Lyrae stars are therefore expected.

We note that the limiting radius 45 arcsec adopted for the membership discussion is significantly smaller than the cluster tidal radius. In fact, many stars outside this arbitrary chosen limiting radius may well individually belong to the cluster.

\section{Oosterhoff type and metallicity}

The pulsation properties of the For 5 RR Lyrae stars are summarized in Table 5 where we list the total number of variable stars with well established type classification (Ntot), the number of fundamental mode (Nab), first overtone ( $\mathrm{Nc}$ ) and double mode pulsators $(\mathrm{Nd})$, the ratio of number of RRc to total number of RR Lyrae stars, the average periods of the $a b$ - and c-type RR Lyrae stars, the shortest and longest RRab periods, and the shortest and longest RRc periods.

The average period of the For $5 c$-type RR Lyrae stars is: $\langle P c\rangle=0.356$ days $(\sigma=0.041$ days, average on 11 stars and excluding the double mode pulsator) or $\langle P c\rangle=0.358$ days ( $\sigma=0.040$ days, average on 12 stars $)$ if the double mode pulsator is included. The average period of the $a b$-type RR Lyrae stars is $\langle P a b\rangle=0.590$ days $(\sigma=0.039$ days, average on 13 stars with certain period determination). If we include in the mean also star V20, whose period is uncertain, with two alternative values $P=0.7474$ and 0.769393 days equally possible, the average period becomes: $\langle P a b\rangle=0.601$ days $(\sigma=0.056$ days, average on 14 stars $)$ if we adopt for $\mathrm{V} 20$ the $\mathrm{P}=0.7474$ days period, or $\langle P a b\rangle=0.602$ days $(\sigma=0.061$ days, average on 14 stars $)$ if we adopt for $\mathrm{V} 20$ the $\mathrm{P}=0.769393$ days period. The shortest period RRab is star V14 with $P_{a b, \min }=0.53297$ days, and the longest period RRab is star V20. Here, we have considered only variables within 45 " from the center of For 5 , since they are more likely cluster members (see Sect. 4.4). According to the average period of the fundamental-mode RR Lyrae stars For 5, like For 4 (see Paper I), appears to be an Oosterhoff-intermediate (Oo-Int) cluster, an Oo-type that has no counterpart among the MW GC\&2, but rather common instead among

\footnotetext{
${ }^{2}$ We recall that in the MW, GCs that contain significant numbers of RR Lyrae stars divide into two distinct groups according to the average periods of the fundamental-mode and first-overtone pulsators, and the fraction of first-overtone over total number of RR Lyrae stars $\left(\mathrm{f}_{\mathrm{c}}\right)$. Namely, OoI clusters have $\langle P a b\rangle=0.55$,
} 
the extragalactic clusters (Catelan 2004, 2009). We thus confirm, with improved reliability, the cluster Oosterhoff classification suggested by MG03.

The period-amplitude distributions of the For 5 RR Lyrae stars is shown in Fig. 6, where expanded symbols are used for stars located at distances less than $45^{\prime \prime}$ from the center of For 5. Figure 6 shows that the majority of the $a b$-type RR Lyrae stars in For 5 appears to be on the OoI line; however, a number of RRab's are found near the OoII location. It is also noteworthy that the average period of the first-overtone pulsators is more similar to those of the OoII Galactic GCs. Furthermore, if we restrict ourselves to variables with more secure membership, the ratio of first-overtone pulsators (RRc) over total number of RR Lyrae stars $\left(\mathrm{f}_{\mathrm{c}}=0.41 / 0.44\right)$ is closer to the typical value of an OoII cluster.

The metallicity of For 5 is somewhat uncertain, being $[\mathrm{Fe} / \mathrm{H}]=-2.20 \pm 0.20$ dex for Buonanno et al. (1998), and $[\mathrm{Fe} / \mathrm{H}]=-1.73 \pm 0.13$ dex for Strader et al. (2003), on the Zinn \& West (1984) metallicity scale, and a systematic difference of 0.47 dex between the two independent studies. To get some hint of the cluster metallicity we have fit the CMD of For 5 to the mean ridgelines of the Galactic globular clusters M15 (NGC7078; $[\mathrm{Fe} / \mathrm{H}]_{\mathrm{M} 15}=$ $-2.15 \pm 0.08$, Zinn \& West 1984), and M3 (NGC5272; $[\mathrm{Fe} / \mathrm{H}]_{\mathrm{M} 3}=-1.66 \pm 0.06$, Zinn \& West 1984), taken from Durrell \& Harris (1993) for M15 and Ferraro et al. (1997) for M3, shifted in magnitude and color to match the For 5 CMD main branches. This comparison is shown in Fig. 1, where the red solid line is the ridgeline of M15 and the blue dashed line is the ridgeline of M3. To match the For 5 CMD we have shifted the M15 ridgeline by $\Delta V=+5.50 \mathrm{mag}$ in magnitude, and $\Delta(B-V)=-0.05 \mathrm{mag}$ in color. Since the reddening of M15 is $E(B-V)_{\mathrm{M} 15}=0.10 \pm 0.01 \mathrm{mag}$ (Durrell \& Harris 1993), the color shift required to match For 5 to M15 implies for the cluster a reddening $E(B-V)_{\text {For } 5}=0.05 \pm 0.01 \mathrm{mag}$, in good agreement with the value derived from the color of the blue edge of the RR Lyrae instability strip (see Sect. 4.2). Similarly, M3 $\left(E(B-V)_{\mathrm{M} 3}=0.01 \pm 0.01 \mathrm{mag}\right.$, Harris 1996) was shifted by $\Delta(B-V)=+0.04 \mathrm{mag}$ in color to match the reddening of For 5 , and by $\Delta V=+5.70 \mathrm{mag}$ in magnitude. The CMD of For 5 is reproduced by the ridgeline of M15, while the M3 ridgeline is about $0.1 \mathrm{mag}$ too red than that of For 5. This comparison suggests that the metal abundance of For 5 is closer to the metallicity of M15 and lower than that of M3, in agreement with the conclusions of Buonanno et al. (1998).

Metallicity estimates can also be derived from the cluster RR Lyrae stars, by using a variety of methods either based on the shape of the light curve of individual stars, or on the mean pulsation properties of the sample. We have used the parameters of the Fourier

$\langle P c\rangle=0.32, \mathrm{f}_{\mathrm{c}}=0.17$, while OoII clusters have $\langle P a b\rangle=0.64,\langle P c\rangle=0.37$, and $\mathrm{f}_{\mathrm{c}}=0.44$ (Oosterhoff 1939, Clement et al. 2001). 
decomposition of the $V$-band light curves, along with Jurcsik \& Kovács (1996) method for the RRab stars (equation 3 in their paper), and Morgan, Wahl \& Wieckhorst (2007) formula for the RRc stars (equation 3 in their paper), to estimate individual metallicities, with accuracy of about 0.3 dex, for the For 5 RR Lyrae stars which have well sampled and regular light curves. Results are summarized in Table 6. The corresponding average metal abundance, for stars within $45^{\prime \prime}$ from the cluster center, is: $\langle[\mathrm{Fe} / \mathrm{H}]\rangle=-1.97 \operatorname{dex}(\sigma=0.16$, average on 9 stars), on the Zinn \& West (1984) metallicity scale. Using instead relations from Sandage (2006), we find $[\mathrm{Fe} / \mathrm{H}]=-2.10$ dex from the mean period of the For 5 fundamental mode RR Lyrae stars $(\langle P a b\rangle=0.590$ days $)$; and -2.33 dex from the shortest period RRab, V14, a star located within $45^{\prime \prime}$ from the cluster center. Finally, following Sandage (1993), we find $[\mathrm{Fe} / \mathrm{H}]=-1.86 /-1.88$ dex, from the mean period of the RRc/RRd stars within $45^{\prime \prime}$ from the cluster center. All these determinations are on the Zinn\& West (1984) metallicity scale. Thus, different methods based on the cluster RR Lyrae stars seem to suggest a low metal abundance for For 5, closer to that of Buonanno et al. (1998).

Nevertheless, we note that none of the conclusions on the Oo-Int status of For 5 are affected in any way by a change in the cluster metal abundance from -2.2 to -1.7 dex since, independently of the adopted metal abundance, the $\langle P a b\rangle$ value places For 5 in the Oo-Int region of the $\left\langle P_{a b}\right\rangle-[\mathrm{Fe} / \mathrm{H}]$ diagram (Pritzl et al. 2002; Catelan 2009). In particular, if $[\mathrm{Fe} / \mathrm{H}]=-1.7$ dex as derived by Strader et al. (2003), For 5 would fall right in the middle of the distribution of the Oo-Int clusters, whereas adopting $[\mathrm{Fe} / \mathrm{H}]=-2.2$ dex actually places the cluster slightly to the right of this distribution (see Figs. 6 and 9 of Catelan 2009). In fact, and as discussed in Catelan et al. (2009, in preparation), the key quantities defining Oosterhoff status appear to be the average and minimum periods of the $a b$-type RR Lyrae stars. In terms of these two quantities For 5 appears entirely consistent with an Oo-Int classification.

We have also reconsidered the HB morphology of For 5 by computing the Lee-Zinn parameter (Zinn 1986; Lee 1990; Lee, Demarque \& Zinn 1990), $(B-R) /(B+V+R)$ (where $B, R$, and $V$ represent the numbers of blue, red, and variable stars on the HB, respectively) of For 5, based on our photometry and number counts. We have considered only stars with $8^{\prime \prime}<r<45^{\prime \prime}$, since we do not have reliable photometry of the Magellan data within $8^{\prime \prime}$. Our results are: $R=8 \pm 1, V=16 \pm 1, B=33 \pm 1$, and $(B-R) /(B+V+R)=0.44 \pm 0.17$ in agreement with Buonanno et al. (1998) who derived 0.44 \pm 0.09 , and slightly smaller than the MG03 values, who find $0.52 \pm 0.04$ (all chips) and $0.52 \pm 0.05$ (PC only). According to our $(B-R) /(B+V+R)$ value For 5 locates on the right edge of the distribution of the Oo-Int clusters band in the $[\mathrm{Fe} / \mathrm{H}]-\mathrm{HB}$ type diagram (see Fig. 8 in Catelan 2009), for $[\mathrm{Fe} / \mathrm{H}]=-2.20$ dex, and on the middle of this band for $[\mathrm{Fe} / \mathrm{H}]=-1.73$ dex. We note that the MG03 values would instead place For 5 slightly outside the Oo-Int band for $[\mathrm{Fe} / \mathrm{H}]=-2.20$ dex (see Fig. 
8 of Catelan 2009).

The properties of the RR Lyrae star populations in the Fornax dSph GCs are summarized in Table 7. Results for For 5 from the present work, and for For 4 from Paper I, are reported in the upper portion of the table. They correspond to variable stars whose membership to the parent clusters is more certain and contamination by field stars is minimized. Results for For 5 from MG03 are reported instead in the lower part of the table for comparison with the present study. In the last three rows of the table we also summarize the MG03 results for For 1, For 2 and For 3. We will revise and update the numbers for these three clusters in the following papers of this series. There is generally good agreement between our results for For 5 and those of MG03, when for the latter, we restrict to variable stars which MG03 consider to have good period estimates. Some differences are found instead among the parameters of the fundamental-mode RR Lyrae stars derived in the two studies, since, as discussed in Sect. 4.3, MG03 generally tend to underestimate the period of the $a b$-type RR Lyrae stars. MG03 $\mathrm{f}_{\mathrm{c}}$ value based on all the candidate RR Lyrae stars differs largely from the value derived using only variables which MG03 consider to have good periods; however, it is interesting that our $\mathrm{f}_{\mathrm{c}}$ values are closer to the value based on the full sample of candidate RR Lyrae stars that MG03 found in the cluster.

The globular clusters of the Fornax dSph appear all to have an Oosterhoff intermediate type, based on the mean period of their $a b$-type RR Lyrae stars, $\left\langle P_{a b}\right\rangle$ (see Col. 3 of Table 7). Indeed, they are confined within the Oosterhoff 'gap' and span it almost entirely from its short period edge (with For 2) to its upper period edge (with For 3). These 5 globulars form a fairly homogeneous sample of Oosterhoff intermediate clusters and may contribute to unveiling the nature of the Oosterhoff intermediate type, namely whether it is just a transition class or whether it is a distinct group with physical properties common to all OoInt clusters (see Catelan et al. 2009). However, the results for the Fornax clusters are not straightforward. We note that the mean periods of the c-type RR Lyrae stars in these clusters are more similar to those of OoII Galactic clusters, and are generally longer than those of the Oosterhoff intermediate globular clusters in the LMC. On the other hand, the full range of $f_{c}$ values from OoI to OoII types is spanned by these clusters, thus suggesting a weak reliability of this parameter to discriminate among Oosterhoff types. The period-amplitude diagrams of the Fornax dSph clusters do not provide clearcut indications either, since in this plane the Fornax clusters show a variety of positions ranging from Oo I (e.g. For 2, and For 4) to Oo-Int/Oo II types, thus suggesting that the Oosterhoff intermediate status is not necessarily accompanied by a clearcut intermediate (e.g., between OoI and Oo I types) position of the RR Lyrae stars in the Bailey diagram. Rather, the Fornax clusters seem to support the idea that the position of the blue edge for first overtone pulsation $\left(P_{\mathrm{ab}, \mathrm{min}}\right)$ plays a dominant role in defining the Oosterhoff intermediate status. We will come back to this 
issue in Catelan et al. (2009).

\section{Cluster Distances}

The average magnitude of the RR Lyrae stars in For 5 is $\langle V(\mathrm{RR})\rangle=21.35 \pm 0.02 \mathrm{mag}$ ( $\sigma=0.07 \mathrm{mag}$, average on 14 stars with well sampled light curves and more secure membership to the cluster). This average value changes only marginally if we also include in the sample 7 RR Lyrae stars in the central region of For 5 which only have HST photometry (see upper portion of Table 3) becoming $\langle V(\mathrm{RR})\rangle=21.34 \pm 0.02 \mathrm{mag}$ ( $\sigma=0.09 \mathrm{mag}$, average on 21 stars). Our $\langle V(\mathrm{RR})\rangle$ values agree within their uncertainties with the Buonanno et al. (1998) apparent magnitude of the cluster $\mathrm{HB}, V_{\mathrm{HB}}($ For 5$)=21.30 \pm 0.05$, and with the average magnitude of the full sample of candidate RR Lyrae stars (40 stars) detected in For 5 by MG03, $\langle V(\mathrm{RR})\rangle=21.33 \pm 0.01$.

In order to derive the distance to For 5 from the average luminosity of the RR Lyrae stars we need estimates of the cluster reddening and metallicity, as well as values for the slope and zero point of the RR Lyrae luminosity-metallicity relation. For consistency with the analysis of For 4 in Paper I we adopt an absolute magnitude of $M_{V}=0.59 \pm 0.03$ mag for RR Lyrae stars of $[\mathrm{Fe} / \mathrm{H}]=-1.5$ dex (Cacciari \& Clementini, 2003), and $\Delta M_{V} /[\mathrm{Fe} / \mathrm{H}]=0.214 \pm 0.047$ $\mathrm{mag} / \mathrm{dex}$ (Clementini et al. 2003, Gratton et al. 2004) for the slope of the luminositymetallicity relation. We then adopt a standard extinction law $\left(\mathrm{A}_{V}=3.1 \times E(B-V)\right)$ and a reddening value of $0.05 \pm 0.01 \mathrm{mag}$, which was derived as the weighted average of four independent estimates, namely: (i) the $E(B-V)$ value of MG03, $E(B-V)=0.03 \pm 0.01$ (intrinsic) \pm 0.02 (systematic) mag; (ii) the Buonanno et al.(1998) $E(V-I)$ transformed to $E(B-V), E(B-V)=0.06 \pm 0.05$ mag; (iii) the reddening we have obtained by matching the cluster CMD to the mean ridgelines of M15, $E(B-V)=0.05 \pm 0.01$ mag (see Sect. 5); and (iv) the reddening we derive by matching the blue edge of the RR Lyrae strip in M3 to the colors of the bluest RR Lyrae stars in the cluster, $E(B-V)=0.04 \pm 0.03$ mag (see Sect. 4.2). The distance modulus of For 5 is then $\mu_{0}($ For 5$)=20.76 \pm 0.07\left(d=141.9_{-4.5}^{+4.6} \mathrm{kpc}\right)$ if we adopt for the cluster the metal abundance by Buonanno et al. $(1998 ;[\mathrm{Fe} / \mathrm{H}]=-2.20 \pm 0.20)$, or $\mu_{0}($ For 5$)=20.66 \pm 0.07\left(d=135.5_{-4.3}^{+4.4} \mathrm{kpc}\right)$ if we adopt the Strader et al. (2003) metallicity $([\mathrm{Fe} / \mathrm{H}]=-1.73 \pm 0.13)$. Errors in the cluster distance moduli are the sum in quadrature of uncertainties of 0.02 in $\langle V(\mathrm{RR})\rangle$ (dispersion of the average), 0.05 mag in the zero point of the photometry, $0.01 \mathrm{mag}$ in $E(B-V)$ (corresponding to $0.03 \mathrm{mag}$ in $A_{V}$ ), and of 0.03 mag and $0.047 \mathrm{mag} / \mathrm{dex}$, respectively, in the zero point and in the slope of the RR Lyrae luminosity-metallicity relation. The cluster distance modulus does not change significantly if we use a different RR Lyrae luminosity-metallicity relation, e.g. that of Chaboyer (1999) 
or that of Catelan \& Cortés (2008).

Distances to the Fornax dSph GCs from the present study and from Paper I for For 4 are summarized in Table 8 and compared with distances from the MG03 study. Our distance moduli for For 5 compare very well with the MG03 estimates particularly if the low metallicity value is adopted for the cluster.

\section{Summary and conclusions}

We have obtained light curves and periods for 30 RR Lyrae stars (13ab-, $14 c$ - and $1 d$ type pulsator) and 1 SX Phe variable in the globular cluster For 5 of the Fornax dSph galaxy. According to our estimate of the surface density of RR Lyrae stars in the field of the Fornax dSph surrounding For 5, at least $28 \pm 1$ out of 30 RR Lyrae variables are cluster members. The average periods of $a b$ - and $c$-type RR Lyrae stars, and the minimum period of the $a b$ type pulsators point to an Oosterhoff-intermediate status for the cluster, unlike what is seen for the vast majority of the Galactic globular clusters. The previous Oosterhoff-intermediate classification suggested by MG03 is confirmed with improved reliability. Our photometry and number counts also suggest a value of the Lee-Zinn HB type of $(B-R) /(B+V+R)$ $=0.44 \pm 0.17$ for For 5 . This value places the cluster on the Oo-Int clusters band in the $[\mathrm{Fe} / \mathrm{H}]$-HBtype diagram.

The average magnitude of the RR Lyrae stars that are more likely members of For 5 is $\langle V(\mathrm{RR})\rangle=21.35 \pm 0.02 \mathrm{mag}$, and leads to $\mu_{0}=20.76 \pm 0.07 \mathrm{mag}$ or $\mu_{0}=20.66 \pm 0.07 \mathrm{mag}$ whether Buonanno et al. $[\mathrm{Fe} / \mathrm{H}]=-2.20$ dex, or Strader et al. $[\mathrm{Fe} / \mathrm{H}]=-1.73$ dex, are adopted for the cluster metallicity. The pulsation properties of the RR Lyrae stars support a low metal abundance for For 5 closer to the Buonanno et al. value, and the distance modulus of $\mu_{0}=20.76 \pm 0.07 \mathrm{mag}\left(d=141.9_{-4.5}^{+4.6} \mathrm{kpc}\right)$. This distance modulus for the cluster agrees well with the value found by MG03.

We thank the anonymous referee for comments. This research was funded by PRIN INAF 2006 (P.I.: G. Clementini). Support for M.C. is provided by Proyecto Basal PFB06/2007, by FONDAP Centro de Astrofísica 15010003, by Proyecto FONDECYT Regular \#1071002, and by a John Simon Guggenheim Memorial Foundation Fellowship. HAS thanks the National Science Foundation for support under grant AST 0607249. 


\section{REFERENCES}

Alard, C. 2000, A\&AS, 144, 363

Barning, F.J.M. 1963, Bull. Astron. Inst. Netherlands, 17, 22

Buonanno, R., Corsi, C. E., Fusi Pecci, F., Hardy, E., \& Zinn, R. 1985, A\&A, 152, 65

Buonanno, R., Corsi, C. E., Zinn, R., Fusi Pecci, F., Hardy, E., \& Suntzeff, N. B. 1998, ApJ, 501, L33

Chaboyer, B. 1999, in Post-Hipparcos cosmic candles, ed. A. Heck and F. Caputo (Dordrecht; Boston : Kluwer Academic Publishers), Astrophysics and space science library, 237, 111

Cacciari, C., \& Clementini, G. 2003, in Stellar Candles for the Extragalactic Distance Scale, ed. D. Alloin and W. Gieren (Berlin: Springer, LNP), Lecture Notes in Physics, 635, 105

Cacciari, C., Corwin, T.M., \& Carney, B.W. 2005, AJ, 129, 267

Catelan, M. 2004, in Variable Stars in the Local Group, ed. D. W. Kurtz \& K. R. Pollard, ASP ASP Conf. Ser., 113

Catelan, M. 2009, Ap\&SS, 320, 261

Catelan, M., \& Cortés, C. 2008, ApJ, 676, L135

Clement, C. M., \& Rowe, J. 2000, AJ, 120, 2579

Clement, C.M., et al. 2001, AJ, 122, 2587

Clementini, G., et al. 2000, AJ, 120, 2054

Clementini, G., Gratton, R., Bragaglia, A., Carretta, E., Di Fabrizio, L., \& Maio, M. 2003, AJ, 125, 1309

Clementini, G., et al. 2006, Mem.S.A.It., 77, 249

Demers, S., Irwin, M.J., \& Kunkel, W.E. 1994, AJ, 108, 1648

Di Fabrizio, L., 1999, Laurea Thesis, Università degli Studi di Bologna

Dubath, P., Meylan, G., \& Mayor, M. 1992, ApJ, 400, 510 
Durrell, P.R., \& Harris, W.E. 1993, AJ, 105, 1420

Ferraro, F.R., Carretta, E., Corsi, C.E., Fusi Pecci, F., Cacciari, C., Buonanno, R., Paltrinieri, B., \& Hamilton, D. 1997, A\&A, 320, 757

Gratton, R.G., Bragaglia, A., Clementini, G., Carretta, E., Di Fabrizio, L., Maio, M., \& Taribello, E. 2004, A\&A, 421, 937

Greco, C., et al. 2007, ApJ, 670, 332 (Paper I)

Hardy, E. 2002, in IAU Symp. 207, Extragalactic Star Clusters, ed. D. Geisler, E. Grebel, \& D. Minniti (San Francisco:ASP), 62

Harris, W.E. 1996, AJ, 112, 1487

Hodge, P. W. 1961, AJ, 66, 83

Hodge, P. W. 1965, ApJ, 141, 308

Hodge P. W. 1969, AJ, 720, 249

Jurcsik, J. 1995, AcA, 45, 653

Jurcsik, J., \& Kovács, G. 1996, A\&A, 312, 111

Landolt, A.U. 1992, AJ, 104, 340

Lee, Y.-W 1990, ApJ, 363, 159

Lee, Y.-W, Demarque, P., \& Zinn, R. 1990, ApJ, 350, 155

Lomb, N.R. 1976, Ap\&SS, 39, 447

Mackey, A. D., \& Gilmore, G. F. 2003a, MNRAS, 340, 175

Mackey, A. D., \& Gilmore, G. F. 2003b, MNRAS, 345, 747

Morgan, S.M., Wahl, J.N., \& Wieckhorst, R.M. 2007, MNRAS, 374, 1421

Oosterhoff, P.Th. 1939, Observatory, 62, 104

Poretti, E., et al. 2008, ApJ, 685, 947 (Paper II)

Pritzl, B. J., Smith, H.A., Catelan, M., \& Sweigart, A.V. 2002, AJ, 124, 949; erratum: 2003, AJ, 125,2752 
Ripepi, V., et al. 2004, CoAst, 145, 24

Sandage, A. 1993, AJ, 106, 687

Sandage, A. 2006, AJ, 131, 1750

Scargle, J.D. 1982, ApJ, 263, 835

Schlegel, D.J., Finkbeiner, D.P., \& Davis, M. 1998, ApJ, 500, 525

Smith, E.O., Neill, J.D., Mighell, K.J., \& Rich, R.M. 1996, AJ, 111, 1596

Stetson, P. B. 1994, PASP, 106, 250

Strader, J., Brodie, J.P., Forbes D.A., Beasley M.A., \& Huchra, J.P. 2003, AJ, 125, 1291

Webbink, R.F. 1985, in Goodman, J., Hut, P., eds, Proc. IAU Symp. 113, Dynamic of star clusters, Kluwer, Dordrecht, p.541

Zinn, R. 1986, in Stellar Populations, ed. C.A. Norman, A. Renzini, \& M. Tosi (Cambridge University press), 73

Zinn, R., \& West, M. J. 1984, ApJS, 55, 45

This preprint was prepared with the AAS LATEX macros v5.2.

Table 1: Instrumental Set-up and Log of the Observations

\begin{tabular}{clllll}
\hline \hline $\begin{array}{l}\text { Dates } \\
(\mathrm{UT})\end{array}$ & Telescope & Instrument & $\mathrm{N}_{\mathrm{B}}$ & $\mathrm{N}_{\mathrm{V}}$ & $\mathrm{N}_{\mathrm{I}}$ \\
\hline 1996, June $4-5$ & HST & WFPC2/PC & $\ldots$ & 14 & 16 \\
& & WFPC2/WF & $\ldots$ & 14 & 16 \\
2003, Nov.13 - 15 & Magellan/Clay & MagIC & 20 & 52 & $\cdots$ \\
\hline
\end{tabular}


Table 2: $V, B$ Photometry of the For 5 Variable Stars with Light Curves on a Magnitude Scale

\begin{tabular}{cccc}
\hline \hline \multicolumn{4}{c}{ For 5 - Star V19 - RRc } \\
\hline $\begin{array}{c}\text { HJD } \\
(-2450238)\end{array}$ & $\begin{array}{c}\mathrm{V} \\
(\mathrm{mag})\end{array}$ & $\begin{array}{c}\text { HJD } \\
(-2452956)\end{array}$ & $\begin{array}{c}\mathrm{B} \\
(\mathrm{mag})\end{array}$ \\
\hline 0.708973 & 21.51 & 0.546462 & 21.95 \\
0.711751 & 21.55 & 0.551370 & 21.91 \\
0.714529 & 21.52 & 0.579396 & 21.87 \\
0.717307 & 21.54 & 0.584338 & 21.82 \\
0.788839 & 21.53 & 0.608903 & 21.74 \\
0.796246 & 21.57 & 0.613521 & 21.65 \\
0.835948 & 21.45 & 0.618555 & 21.65 \\
0.843587 & 21.32 & 0.643554 & 21.51 \\
0.922873 & 21.34 & 0.691377 & 21.43 \\
0.930281 & 21.31 & 1.555353 & 21.80 \\
\hline
\end{tabular}

Table 2 is published in its entirety in the electronic edition of the Astrophysical Journal. A portion is shown here for guidance regarding its form and content. 
Table 3: Identification and Properties of the For 5 Variable Stars.

\begin{tabular}{|c|c|c|c|c|c|c|c|c|c|c|c|c|c|}
\hline $\begin{array}{l}\text { Name } \\
\qquad(a)\end{array}$ & Id & $\begin{array}{c}\alpha \\
(2000)\end{array}$ & $\begin{array}{c}\delta \\
(2000)\end{array}$ & Type & $\begin{array}{l}\mathrm{P} \\
\text { (days) }\end{array}$ & $\begin{array}{c}\text { Epoch } \\
(-2450000)\end{array}$ & $\begin{array}{c}\langle\mathrm{V}\rangle \\
(\mathrm{mag})\end{array}$ & $\mathrm{N}_{\mathrm{V}}$ & $\begin{array}{c}\langle\mathrm{B}\rangle \\
(\mathrm{mag})\end{array}$ & $\mathrm{N}_{\mathrm{B}}$ & $\begin{array}{c}\mathrm{A}_{\mathrm{V}} \\
(\mathrm{mag})\end{array}$ & $\begin{array}{c}\mathrm{A}_{\mathrm{B}} \\
(\mathrm{mag})\end{array}$ & Notes \\
\hline $\mathrm{V} 1$ & $\mathrm{LC} 117$ & $2: 42: 21.25$ & $-34: 06: 07.60$ & RRab & 0.635 & 2956.587 & $\ldots$ & $54+11$ & $\cdots$ & $\cdots$ & $>0.19$ & $\cdots$ & $(b, c)$ \\
\hline $\mathrm{V} 2$ & LC77 & $2: 42: 21.01$ & $-34: 06: 10.56$ & RRc & 0.4085 & 2957.700 & 21.26 & $55+14$ & $\cdots$ & $\cdots$ & 0.50 & $\cdots$ & $(b)$ \\
\hline V3 & LC103 & $2: 42: 21.40$ & $-34: 06: 08.64$ & RRc & 0.2842 & 2956.623 & 21.33 & $55+14$ & $\ldots$ & $\ldots$ & $\geq 0.37$ & $\ldots$ & (b) \\
\hline V4 & LC166 & $2: 42: 21.34$ & $-34: 06: 03.19$ & RRc & 0.3635 & 2957.797 & 21.21 & $55+14$ & $\ldots$ & $\ldots$ & 0.56 & $\ldots$ & (b) \\
\hline V5 & $\mathrm{LC} 172$ & $2: 42: 21.20$ & $-34: 06: 02.17$ & RRab & 0.56 & $\ldots$ & $\ldots$ & 55 & $\cdots$ & $\cdots$ & $\ldots$ & $\cdots$ & $(b, d)$ \\
\hline V6 & $\mathrm{LC} 175$ & $2: 42: 21.02$ & $-34: 06: 01.68$ & RRab & 0.65 & $\cdots$ & $\ldots$ & $55+11$ & $\cdots$ & $\cdots$ & $\ldots$ & $\cdots$ & $(b, c)$ \\
\hline V7 & LC136 & $2: 42: 20.54$ & $-34: 06: 05.83$ & RRab & 0.605 & $\ldots$ & 21.48 & $55+14$ & $\ldots$ & $\ldots$ & $>0.37$ & $\ldots$ & (b) \\
\hline V8 & LC149 & $2: 42: 21.58$ & $-34: 06: 05.01$ & RRab & 0.5620 & 2957.623 & 21.48 & $55+14$ & $\cdots$ & $\cdots$ & $>0.59$ & $\cdots$ & (b) \\
\hline V9 & LC98 & $2: 42: 21.63$ & $-34: 06: 08.83$ & RRab & 0.6310 & 2956.593 & 21.23 & $55+14$ & $\ldots$ & $\ldots$ & $\geq 0.84$ & $\ldots$ & (b) \\
\hline V10 & LC118 & $2: 42: 21.68$ & $-34: 06: 07.49$ & RRab & 0.6041 & 2956.603 & 21.26 & $51+14$ & $\cdots$ & $\cdots$ & $>0.78$ & $\cdots$ & (b) \\
\hline V11 & 3583 & $2: 42: 20.51$ & $-34: 06: 10.69$ & RRab & 0.547034 & 2957.600 & $21.38:$ & $52+12$ & $21.67:$ & 20 & $>0.76$ & $>0.75$ & \\
\hline V12 & 2577 & $2: 42: 21.67$ & $-34: 06: 04.63$ & RRab & 0.533555 & 238.145 & 21.27 & $51+14$ & 21.52 & 20 & $\geq 0.88$ & $\geq 1.06$ & \\
\hline V13 & 2537 & $2: 42: 21.72$ & $-34: 06: 07.58$ & $\mathrm{RRc}$ & 0.406060 & 237.193 & 21.37 & $50+12$ & 21.68 & 20 & 0.35 & 0.41 & \\
\hline V14 & 2482 & $2: 42: 21.79$ & $-34: 06: 01.59$ & RRab & 0.53297 & $237.615:$ & $21.39:$ & $50+14$ & $21.59:$ & 18 & $\geq 0.56$ & $\geq 0.94$ & \\
\hline V15 & 3845 & $2: 42: 20.21$ & $-34: 06: 05.88$ & $\mathrm{RR}$ & $\ldots$ & $\ldots$ & 21.20 & $47+14$ & 21.54 & 17 & $\geq 0.16$ & $\geq 0.25$ & $(e)$ \\
\hline V16 & 3631 & $2: 42: 20.47$ & $-34: 05: 59.59$ & RRab & 0.605949 & 238.796 & 21.29 & $49+14$ & 21.63 & 20 & 1.06 & 1.22 & \\
\hline V17 & 2252 & $2: 42: 22.05$ & $-34: 06: 07.61$ & RRab & $0.68::$ & $\ldots$ & $21.36:$ & $49+12$ & $21.75:$ & 20 & $>0.23$ & $>0.32$ & $(f)$ \\
\hline V18 & 3274 & $2: 42: 20.87$ & $-34: 06: 20.18$ & $\mathrm{RRc} / \mathrm{d}$ & 0.37023 & 238.720 & 21.37 & $52+14$ & 21.66 & 19 & 0.45 & 0.58 & $(g)$ \\
\hline V19 & 2818 & $2: 42: 21.39$ & $-34: 05: 52.21$ & $\mathrm{RRc}$ & 0.372043 & 2957.782 & 21.36 & $52+14$ & 21.67 & 20 & 0.43 & 0.60 & \\
\hline V20 & 3936 & $2: 42: 20.09$ & $-34: 06: 18.66$ & RRab & $0.7474:$ & 2957.675 & 21.45 & $51+13$ & $21.85:$ & 18 & 0.63 & $\geq 0.74$ & \\
\hline V21 & 2663 & $2: 42: 21.58$ & $-34: 05: 50.29$ & RRc & 0.307246 & 237.875 & 21.40 & $52+14$ & 21.64 & 18 & 0.65 & 0.88 & \\
\hline V22 & 2706 & $2: 42: 21.53$ & $-34: 06: 25.23$ & RRab & 0.61 & 2957.715 & 21.40 & 50 & 21.76 & 19 & 0.69 & 0.84 & $(h)$ \\
\hline V23 & 4097 & $2: 42: 19.86$ & $-34: 05: 56.09$ & RRc & 0.333292 & 237.850 & 21.37 & $47+14$ & 21.61 & 20 & 0.61 & 0.70 & \\
\hline V24 & 1673 & $2: 42: 22.84$ & $-34: 06: 11.51$ & RRc & 0.313763 & 237.685 & 21.35 & $52+13$ & 21.54 & 19 & 0.37 & 0.50 & \\
\hline V25 & 4350 & $2: 42: 19.52$ & $-34: 05: 48.13$ & RRc & 0.365929 & 2957.715 & 21.38 & $50+14$ & 21.65 & 18 & 0.49 & 0.68 & \\
\hline V26 & 5057 & $2: 42: 18.29$ & $-34: 06: 13.03$ & RRc & 0.389 & 2955.858 & 21.28 & 50 & 21.54 & 19 & 0.48 & 0.73 & $(h)$ \\
\hline V27 & 970 & $2: 42: 24.04$ & $-34: 06: 11.83$ & RRab & 0.588055 & 238.152 & $21.30:$ & $52+13$ & $21.65:$ & 20 & $0.99:$ & $>0.63$ & \\
\hline V28 & 4853 & $2: 42: 18.69$ & $-34: 05: 43.30$ & $\mathrm{RRc}$ & 0.3768 & 2957.637 & 21.46 & 51 & 21.76 & 18 & 0.30 & 0.34 & (i) \\
\hline V29 & 3427 & $2: 42: 20.64$ & $-34: 05: 43.59$ & SX Phe & 0.04950 & 2957.755 & 23.74 & 42 & 23.97 & 17 & 0.21 & $0.33(h)$ & \\
\hline V30 & 5472 & $2: 42: 17.46$ & $-34: 07: 16.14$ & RRab & 0.538 & 2956.547 & 21.40 & 52 & 21.68 & 20 & 1.19 & 1.48 & $(h)$ \\
\hline V31 & 157 & $2: 42: 25.64$ & $-34: 05: 07.30$ & RRc & 0.396 & 2957.844 & 21.21 & $49+10$ & 21.36 & 19 & 0.55 & 0.67 & \\
\hline
\end{tabular}

${ }^{a}$ Variable stars were assigned increasing numbers starting from the cluster center, which was set at R.A. $=2^{h} 42^{m} 21.07^{s}, \mathrm{DEC}=-34^{\circ} 06^{\prime} 07.5^{\prime \prime}$, (J2000). Stars from V1 to V28 are located within $45^{\prime \prime}$ from the For 5 center, hence are more likely cluster members (see Sect. 4.4).

b Light curves from the Magellan observations (50-55 data points) are available in differential flux only. Magnitudes, when available are only from the HST dataset (10-14 data points). They were obtained through a rough calibration of the HST F555W data done neglecting the color terms, they provide only a crude estimate of the actual average magnitude of these variable stars, which often have poor light curve coverage in the HST dataset.

c The light curve coverage of V1 and V6 in the HST dataset is not sufficient to provide an estimate of the star average $V$ magnitude.

d V5 has no secure counterpart in the HST photometry.

e Average magnitude and shape of the light curve are consistent with a small amplitude RR Lyrae star, but the period definition is very uncertain. A period of about 0.49 days phases the star light curve, a shorter period around 0.34 days is also possible. MG03 derive for the star a period of 0.394 but with large uncertainty. In Fig. 4 the star light curves were folded according to the period $\mathrm{P}=0.34$ which is closer to the value found by MG03.

${ }^{f}$ Shape and amplitude of the light curve indicate a long period fundamental mode RR Lyrae star, but the period definition is very uncertain. A period around 0.68 days phases the light curve well but with a large gap around the minimum light. Longer periods around 0.73 or 0.79 days are also possible.

$\mathrm{g}$ The scatter and specifically the two maxima slightly different in shape observed in the light curves of V18 in two consecutive nights suggest that the star could be a double-mode RR Lyrae variable.

$\mathrm{h}$ These stars fall outside the FOV covered by the HST observations.

i The HST photometry of V28 is systematically brighter than the Magellan photometry. 
Table 4: Cross-identification with MG03 study of the variable stars in For 5.

\begin{tabular}{lllllllc}
\hline \hline Name & Id & HST Camera & Type & P & Id & Type & P \\
This paper & This paper & & This paper & $\begin{array}{l}\text { This paper } \\
\text { (days) }\end{array}$ & MG03 & MG03 & $\begin{array}{c}\text { MG03 } \\
\text { (days) }\end{array}$ \\
& & & & & & \\
V2 & LC77 & PC & RRc & 0.4085 & F5V15 & RRc & 0.362 \\
V3 & LC103 & PC & RRc & 0.2842 & F5V03 & RRc & 0.278 \\
V4 & LC166 & PC & RRc & 0.3635 & F5V11 & RRc & 0.364 \\
V6 & LC175 & PC & RRab & 0.65 & F5V06 & RRab & 0.595 \\
V7 & LC136 & PC & RRab & 0.605 & F5V38 & RRab & $0.392^{a}$ \\
V8 & LC149 & PC & RRab & 0.5620 & F5V37 & RRab & $0.532^{a}$ \\
V9 & LC98 & PC & RRab & 0.6310 & F5V25 & RRab & 0.494 \\
V10 & LC118 & PC & RRab & 0.6041 & F5V10 & RRc & 0.340 \\
V11 & 3583 & PC & RRab & 0.547034 & F5V22 & RRc & 0.402 \\
V12 & 2577 & PC & RRab & 0.533555 & F5V16 & RRab & 0.520 \\
V13 & 2537 & PC & RRc & 0.406060 & F5V29 & RRc & 0.420 \\
V14 & 2482 & PC & RRab & 0.53297 & F5V32 & RRab & 0.603 \\
V15 & 3845 & PC & RR & $\ldots$ & F5V05 & RRc & 0.394 \\
V16 & 3631 & PC & RRab & 0.605949 & F5V26 & RRab & 0.571 \\
V18 & 3274 & PC & RRc/d & 0.37023 & F5V20 & RRc & 0.422 \\
V19 & 2818 & PC & RRc & 0.372043 & F5C02 & $\ldots$ & $\ldots$ \\
V20 & 3936 & PC & RRab & $0.7474:$ & F5V39 & RRab & $0.513^{a}$ \\
V21 & 2663 & PC & RRc & 0.307246 & F5V02 & RRc & 0.311 \\
V23 & 4097 & PC & RRc & 0.333292 & F5V28 & RRc & 0.326 \\
V25 & 4350 & WFPC2 -2 & RRc & 0.365929 & F5V18 & RRc & 0.361 \\
V27 & 970 & WFPC2 -4 & RRab & 0.588055 & F5V30 & RRab & $0.433^{a}$ \\
V28 & 4853 & WFPC2 -2 & RRc & 0.3768 & F5V23 & RRc & 0.379 \\
V31 & 157 & WFPC2 -3 & RRc & 0.396 & F5V12 & RRc & 0.396 \\
\hline & & & & & & \\
\hline
\end{tabular}

${ }^{a}$ Uncertain period determination (MG03).

b Candidate variable according to MG03 Table 3. 
Table 5: Average quantities for the For 5 RR Lyrae stars

\begin{tabular}{|c|c|c|c|c|c|c|c|c|c|c|}
\hline $\begin{array}{l}\text { Ntot } \\
\text { (a) }\end{array}$ & $\begin{array}{l}\mathrm{Nab} \\
\text { (a) }\end{array}$ & $\begin{array}{l}\mathrm{Nc} \\
\text { (a) }\end{array}$ & $\begin{array}{l}\mathrm{Nd} \\
\text { (a) }\end{array}$ & $\begin{array}{c}\text { Nc/Ntot } \\
(a, b, c)\end{array}$ & $\begin{array}{l}\langle\mathrm{Pab}\rangle \\
(\mathrm{a}, \mathrm{d}) \\
(\text { days })\end{array}$ & $\begin{array}{l}\langle\mathrm{Pc}\rangle \\
(\mathrm{a}, \mathrm{e}) \\
(\text { days })\end{array}$ & $\begin{array}{l}\mathrm{P}_{\mathrm{ab}, \mathrm{min}} \\
\text { (days) }\end{array}$ & $\begin{array}{c}\mathrm{P}_{\mathrm{ab}, \max } \\
\text { (days) }\end{array}$ & $\begin{array}{l}\mathrm{P}_{\mathrm{c}, \min } \\
\text { (days) }\end{array}$ & $\begin{array}{l}\mathrm{P}_{\mathrm{c}, \max } \\
\text { (days) }\end{array}$ \\
\hline $29(27)$ & $16(15)$ & $12(11)$ & $1(1)$ & $\begin{array}{l}0.41(0.41) \\
0.45(0.44)\end{array}$ & 0.590 & $\begin{array}{l}0.356 \\
0.358\end{array}$ & 0.53297 & $0.7474:$ & 0.2842 & 0.4085 \\
\hline
\end{tabular}

${ }^{a}$ Numbers in parentheses and the $\left\langle\mathrm{P}_{\mathrm{ab}}\right\rangle,\left\langle\mathrm{P}_{\mathrm{c}}\right\rangle$ values correspond only to RR Lyrae stars whose membership to the clusters is more certain (namely, RR Lyrae stars within $45^{\prime \prime}$ from the cluster center).

b $\mathrm{N}_{\text {tot }}$ is calculated only from RR Lyrae stars with well established type classification (29 out of the 30 RR Lyrae stars in For 5).

c Ratio of RRc to total number of RR Lyrae stars with (2nd row values) and without (1st row values) the candidate RRd star in For 5.

$\mathrm{d}$ The $\left\langle\mathrm{P}_{\mathrm{ab}}\right\rangle$ value of For 5 does not consider the two long period $a b$-type RR Lyrae stars V17 and V20 which have uncertain periods. If these two stars are included $\left\langle\mathrm{P}_{\mathrm{ab}}\right\rangle$ becomes $0.606 / 0.608$ days, depending on the period adopted for V20, whether 0.7474 or 0.769393 days.

e First overtone average period with (2nd row values) and without (1st row values) the candidate double mode star. 
Table 6: Metallicities from the Fourier decomposition of the light curves of the RR Lyrae stars within 45 arcsec from the cluster center.

\begin{tabular}{lllll}
\hline \hline Name & Type & $\begin{array}{c}\mathrm{P} \\
\text { (days) }\end{array}$ & $\phi_{31}$ & {$[\mathrm{Fe} / \mathrm{H}]_{\text {ZW84 }}$} \\
\hline V13 & RRc & 0.406060 & 4.27 & -1.84 \\
V19 & RRc & 0.372043 & 2.77 & -2.11 \\
V21 & RRc & 0.307246 & 1.79 & -2.00 \\
V23 & RRc & 0.333292 & 2.17 & -2.06 \\
V24 & RRc & 0.313763 & 3.03 & -1.66 \\
V25 & RRc & 0.365929 & 3.28 & -2.03 \\
V26 & RRc & 0.389 & 3.22 & -2.07 \\
V27 & RRab & 0.588055 & 1.69 & $-1.81:$ \\
V28 & RRc & 0.3768 & 2.77 & -2.11 \\
\hline
\end{tabular}

The metal abundance of the fundamental mode RR Lyrae star was transformed to the Zinn \& West (1984) metallicity scale by applying the transformation relation provided by equation 4 in Jurcsik (1995).

Table 7: Properties of the RR Lyrae stars in the globular clusters of the Fornax dSph

\begin{tabular}{|c|c|c|c|c|c|c|c|c|c|c|c|}
\hline Cluster & $\begin{array}{c}(\mathrm{B}-\mathrm{R}) /(\mathrm{B}+\mathrm{V}+\mathrm{R}) \\
\text { (a) }\end{array}$ & $\begin{array}{c}\langle\mathrm{Pab}\rangle \\
(\mathrm{b}, \text { days })\end{array}$ & $\begin{array}{c}\langle\mathrm{Pc}\rangle \\
\text { (days) }\end{array}$ & $\begin{array}{l}\mathrm{P}_{\mathrm{ab}, \mathrm{min}} \\
(\mathrm{b}, \text { days })\end{array}$ & $\begin{array}{l}\mathrm{P}_{c, \max } \\
\text { (b, days) }\end{array}$ & $\begin{array}{r}\mathrm{Nab} \\
(\mathrm{c})\end{array}$ & $\mathrm{Nc}$ & $\mathrm{Nd}$ & $\begin{array}{l}f_{c} \\
(d)\end{array}$ & Oo Type & Reference \\
\hline For 5 & 0.44 & 0.590 & $0.356 / 0.358$ & 0.53297 & 0.409 & 15 & 11 & 1 & $0.41 / 0.44$ & Oo - Int & This paper \\
\hline For 4 & -0.75 & 0.594 & 0.360 & 0.5191 & 0.400 & 16 & 3 & 1 & 0.16 & Oo - Int & Paper I \\
\hline For 5 & $0.52(0.52)$ & $0.577(0.532)$ & 0.374 & 0.494 & 0.445 & $8(13)$ & 27 & $\cdots$ & $0.77(0.68)$ & Oo - Int & MG03 \\
\hline For 1 & $-0.30(-0.38)$ & $0.611(0.546)$ & 0.431 & 0.570 & 0.459 & $5(10)$ & 5 & $\cdots$ & $0.50(0.33)$ & Oo - Int & MG03 \\
\hline For 2 & $0.42(0.50)$ & $0.574(0.494)$ & 0.373 & 0.512 & 0.404 & $15(30)$ & 13 & $\ldots$ & $0.46(0.30)$ & Oo - Int & MG03 \\
\hline For 3 & $0.40(0.44)$ & $0.613(0.532)$ & 0.404 & 0.479 & 0.510 & $32(61)$ & 38 & $\ldots$ & $0.54(0.38)$ & Oo - Int & MG03 \\
\hline
\end{tabular}

a $(B-R) /(B+V+R)$ values in parentheses correspond to MG03 counts for stars on the PC only.

$\mathrm{b}$ The $\left\langle\mathrm{P}_{\mathrm{ab}}\right\rangle$ and $\mathrm{P}_{a b, \text { min }}$ values from MG03 are those corresponding only to RRab stars with good periods according to these authors, the values in parentheses are from all the candidate RR Lyrae stars detected in MG03.

c Nab values are the RRab's with good periods for MG03, values in parentheses correspond to the total number of candidate RR Lyrae stars in each cluster, according to the MG03 study.

${ }^{d} f_{c}=\mathrm{Nc} /(\mathrm{N} c+\mathrm{N} a b)$, the $\mathrm{f}_{c}$ values in parentheses correspond to the total number of candidate RR Lyrae stars in each cluster, according to the MG03 study. 
Table 8: Distance moduli for the globular clusters of the Fornax dSph galaxy based on the average magnitude of the RR Lyrae stars

\begin{tabular}{|c|c|c|c|c|c|c|}
\hline Cluster & $\begin{array}{c}\left\langle\mathrm{V}_{\mathrm{HB}}\right\rangle \\
(\mathrm{mag})\end{array}$ & $\begin{array}{l}\mathrm{E}(\mathrm{B}-\mathrm{V}) \\
(\mathrm{mag})\end{array}$ & {$[\mathrm{Fe} / \mathrm{H}]$} & $M_{V} v s[\mathrm{Fe} / \mathrm{H}]$ & $\begin{array}{c}\mu_{0} \\
(\mathrm{mag})\end{array}$ & Reference \\
\hline For 5 & $21.35 \pm 0.02$ & $0.03 \pm 0.01$ & $\begin{array}{l}-2.20 \pm 0.20 \\
-1.73 \pm 0.13\end{array}$ & $0.214 \times([\mathrm{Fe} / \mathrm{H}]+1.5)+0.59$ & $\begin{array}{l}20.76 \pm 0.07 \\
20.66 \pm 0.07\end{array}$ & $\begin{array}{l}\text { This paper; }[\mathrm{Fe} / \mathrm{H}] \text { from Buonanno et al. }(1998) \\
\text { This paper; }[\mathrm{Fe} / \mathrm{H}] \text { from Strader et al. }(2003)\end{array}$ \\
\hline For 4 & $21.43 \pm 0.03$ & $0.10 \pm 0.02$ & $-2.01 \pm 0.20$ & & $20.64 \pm 0.09$ & Paper I \\
\hline For 5 & $21.33 \pm 0.01$ & $0.03 \pm 0.01$ & $-1.90 \pm 0.06$ & $0.23 \times([\mathrm{Fe} / \mathrm{H}]+1.6)+0.56$ & $20.74 \pm 0.05$ & MG03 \\
\hline For 1 & $21.27 \pm 0.01$ & $0.07 \pm 0.01$ & $-2.05 \pm 0.10$ & $0.23 \times([\mathrm{Fe} / \mathrm{H}]+1.6)+0.56$ & $20.58 \pm 0.05$ & MG03 \\
\hline For 2 & $21.34 \pm 0.01$ & $0.05 \pm 0.01$ & $-1.83 \pm 0.07$ & & $20.67 \pm 0.05$ & MG03 \\
\hline For 3 & $21.24 \pm 0.01$ & $0.04 \pm 0.01$ & $-2.04 \pm 0.07$ & & $20.66 \pm 0.05$ & MG03 \\
\hline
\end{tabular}




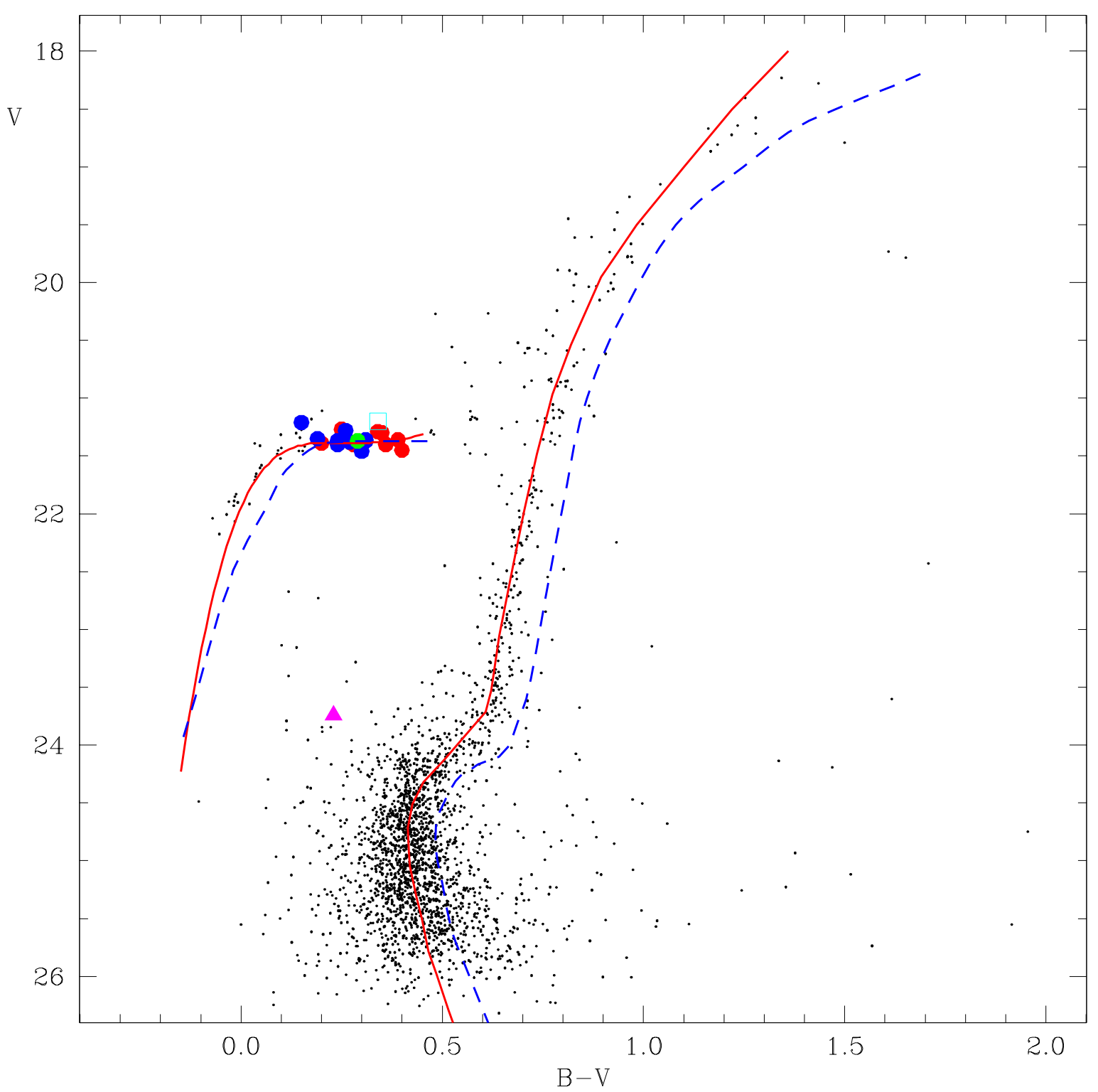

Fig. 1.- Color-magnitude diagram of For 5 from the Magellan data, showing stars located outside an inner region of about $8^{\prime \prime}$ from the cluster center. Variable stars are plotted according to their intensity-averaged magnitudes and colors, using different colors/symbols for the different types. Red circles: ab-type RR Lyrae stars; blue circles: first-overtone RR Lyrae stars; green circle: candidate double-mode pulsator (RRd); square: RR Lyrae star with uncertain type classification; filled triangle: SX Phe star. The bluest and brightest variable is V31, the most external of the For 5 RR Lyrae stars, which may belong to the field of the Fornax dSph. The red (solid) line is the ridgeline of M15 from Durrell \& Harris (1993) and the blue (dashed) line is the ridgeline of M3 from Ferraro et al. (1997). 

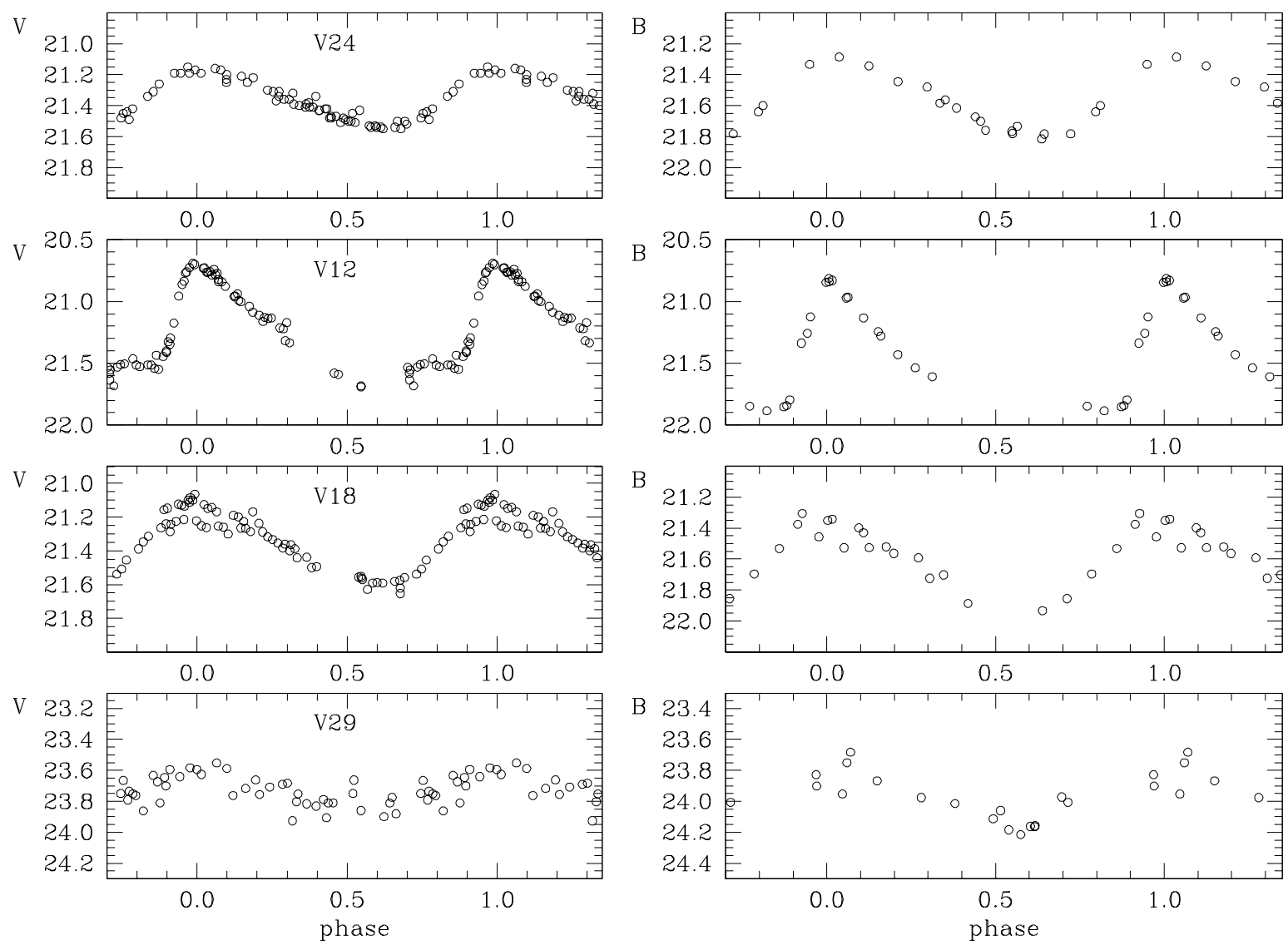

Fig. 2.- $V, B$ light curves of variable stars in For 5. From top to bottom: $c$-, $a b$-, $d$-type RR Lyrae stars, SX Phe star. 

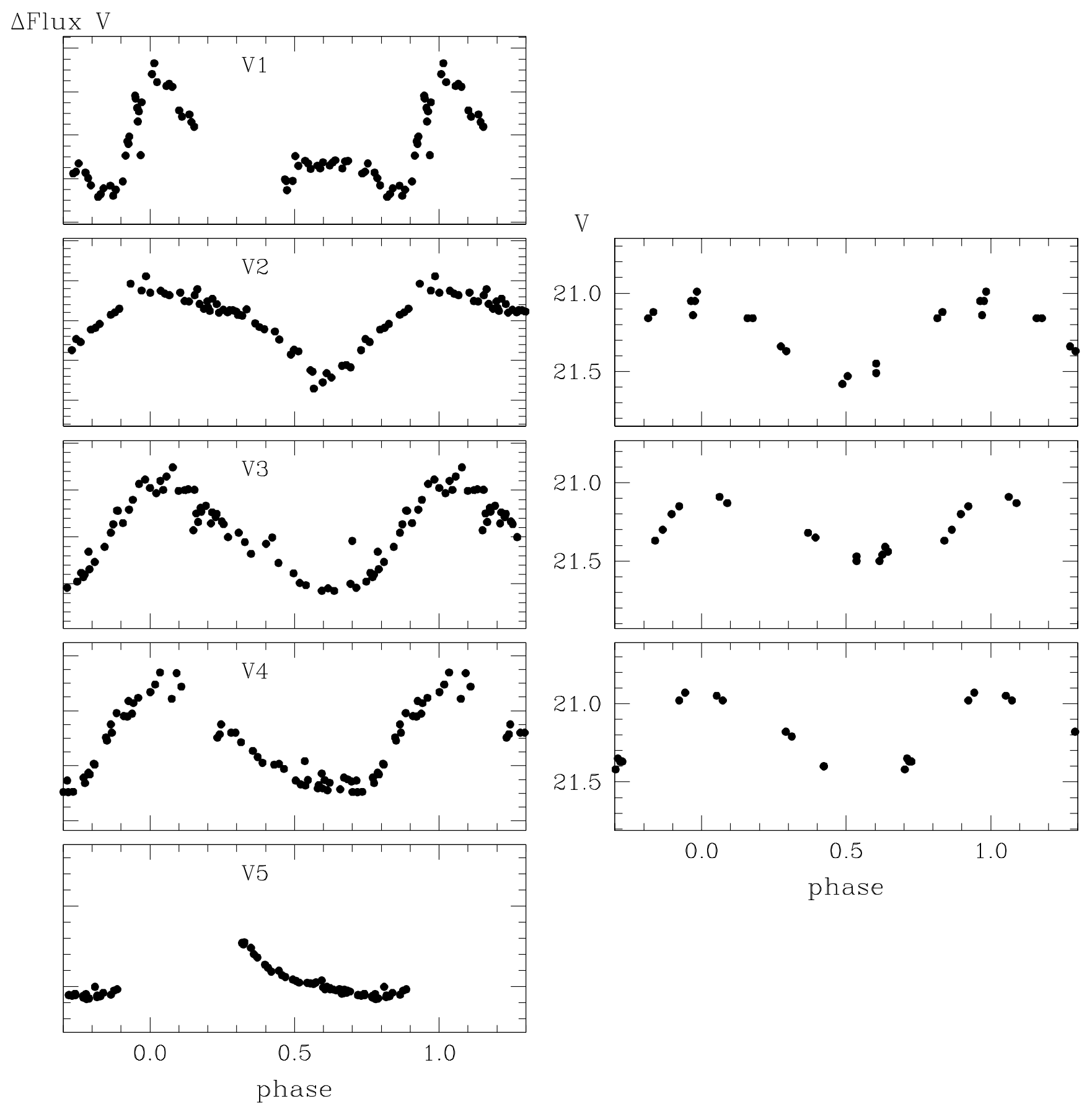

phase

Fig. 3.- Atlas of the light curves for RR Lyrae stars within $8^{\prime \prime}$ from the center of For 5. Left panel: Light curves in $V$ differential flux, from the Magellan dataset. Right panel: Light curves in $V$ magnitude, from the HST dataset. 

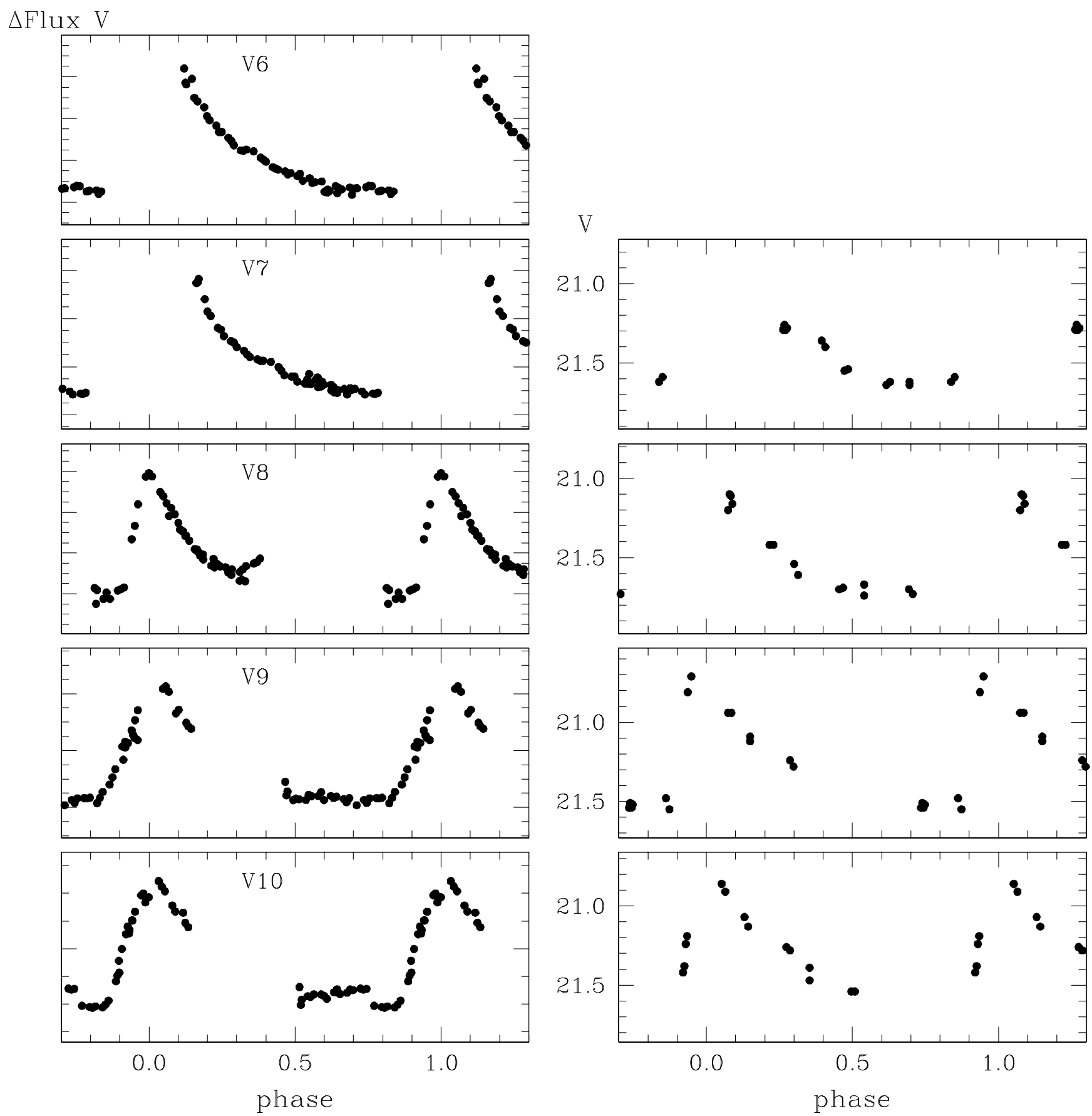

Fig. 3.- continued 
V
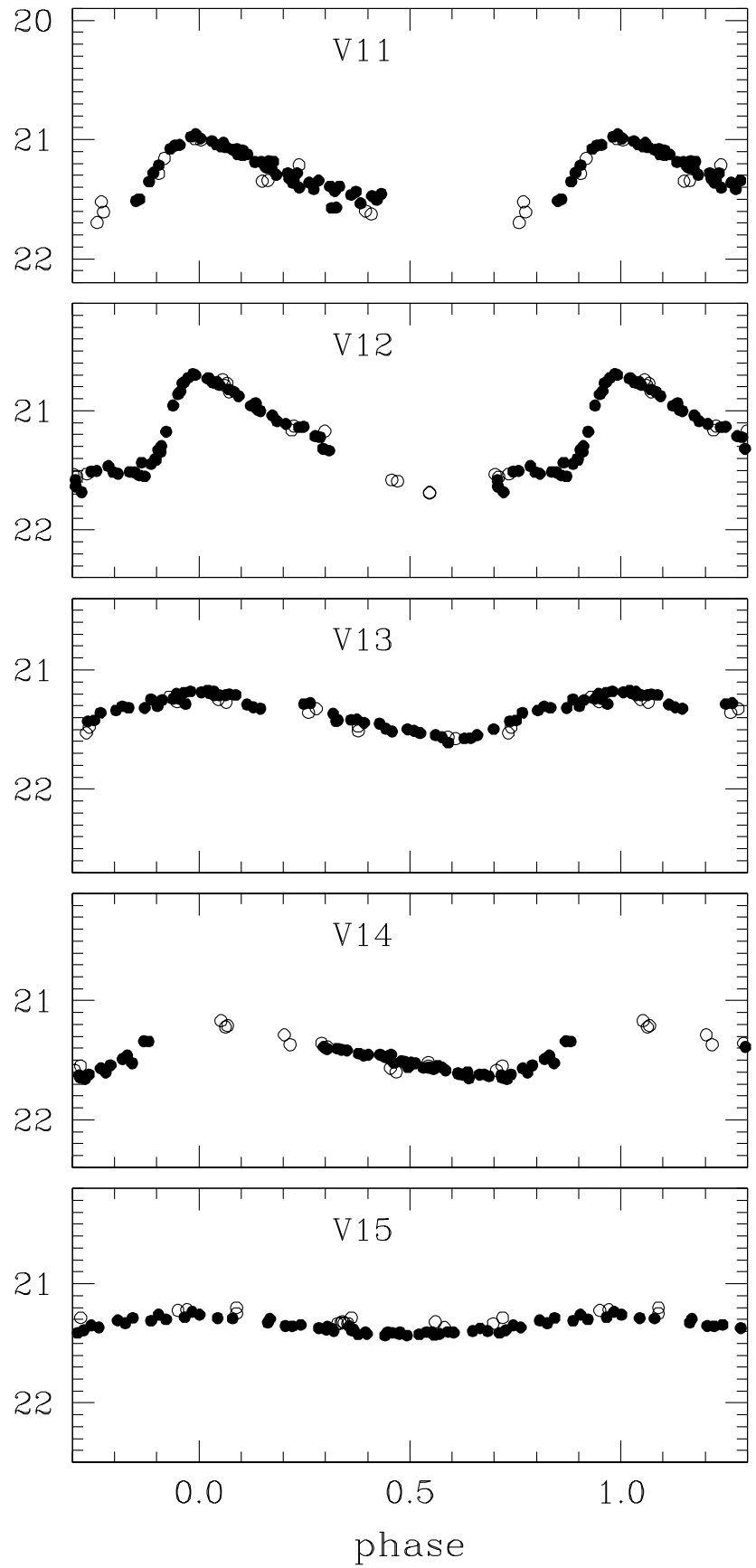

B
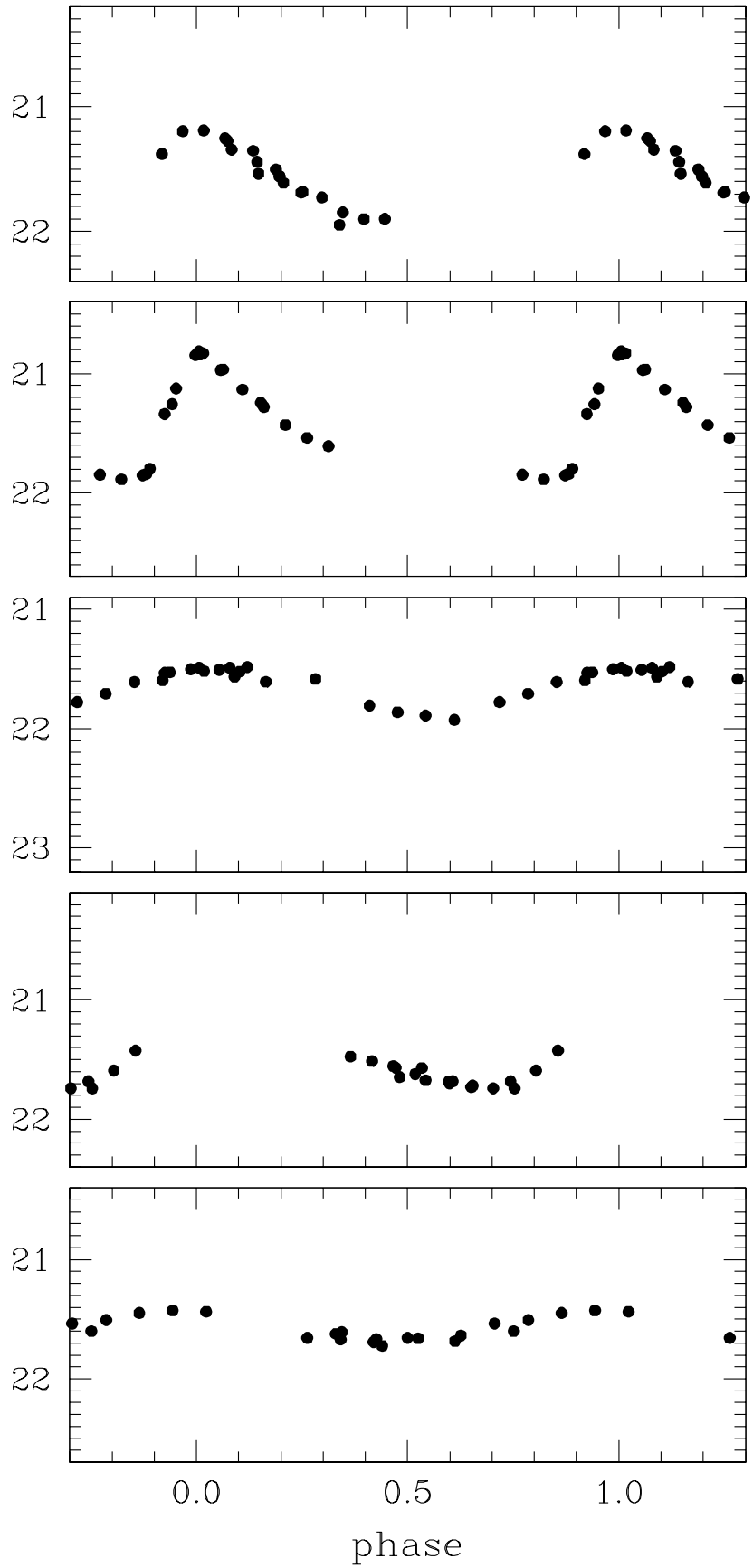

Fig. 4.- Atlas of the $V, B$ light curves of the RR Lyrae stars in For 5 from the combined Magellan (filled circles) and HST (open circles) datasets. Data for star V15 were folded adopting $\mathrm{P}=0.340$ days (see Sect. 4.3). 

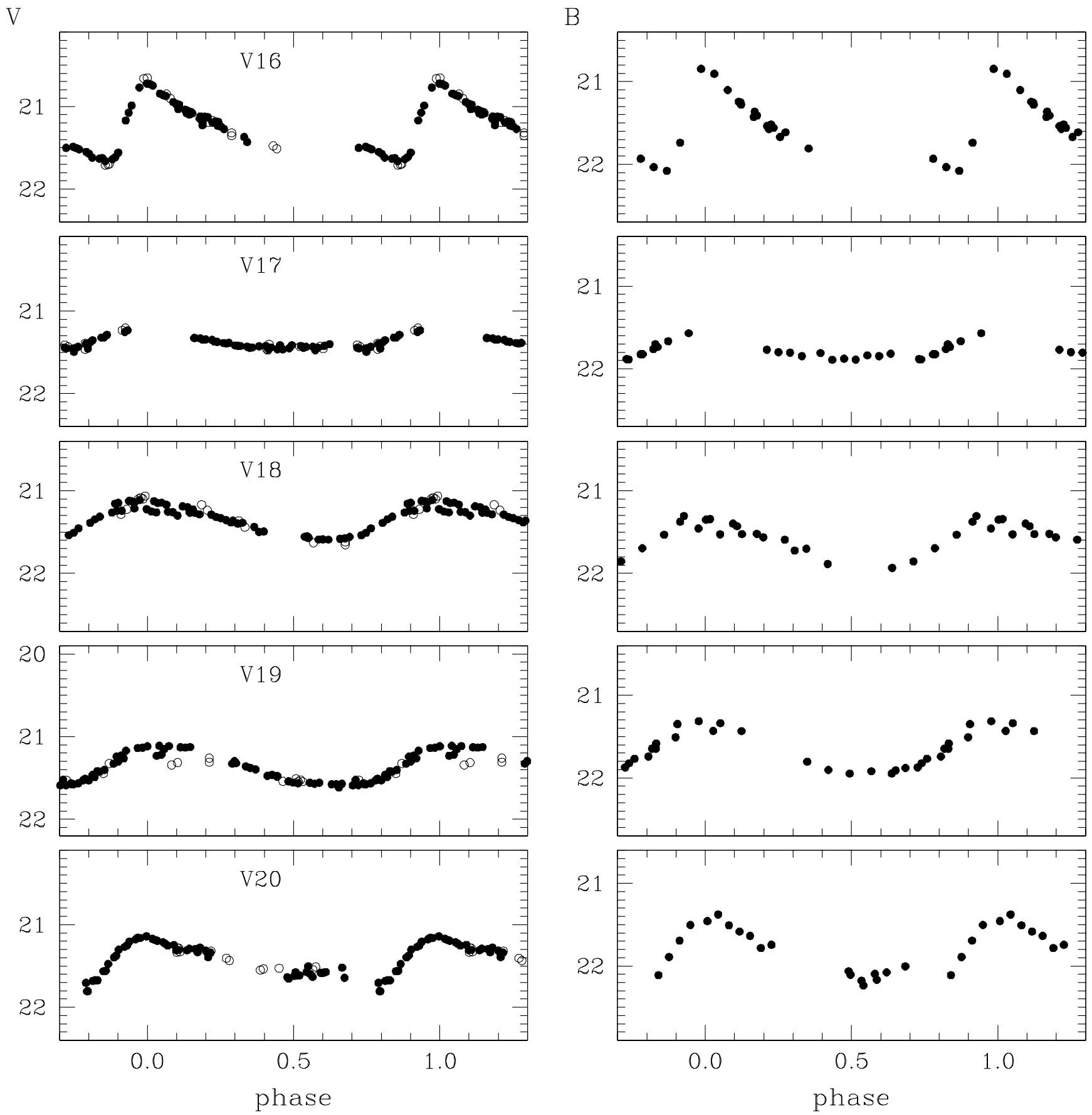

Fig. 4.- continued 

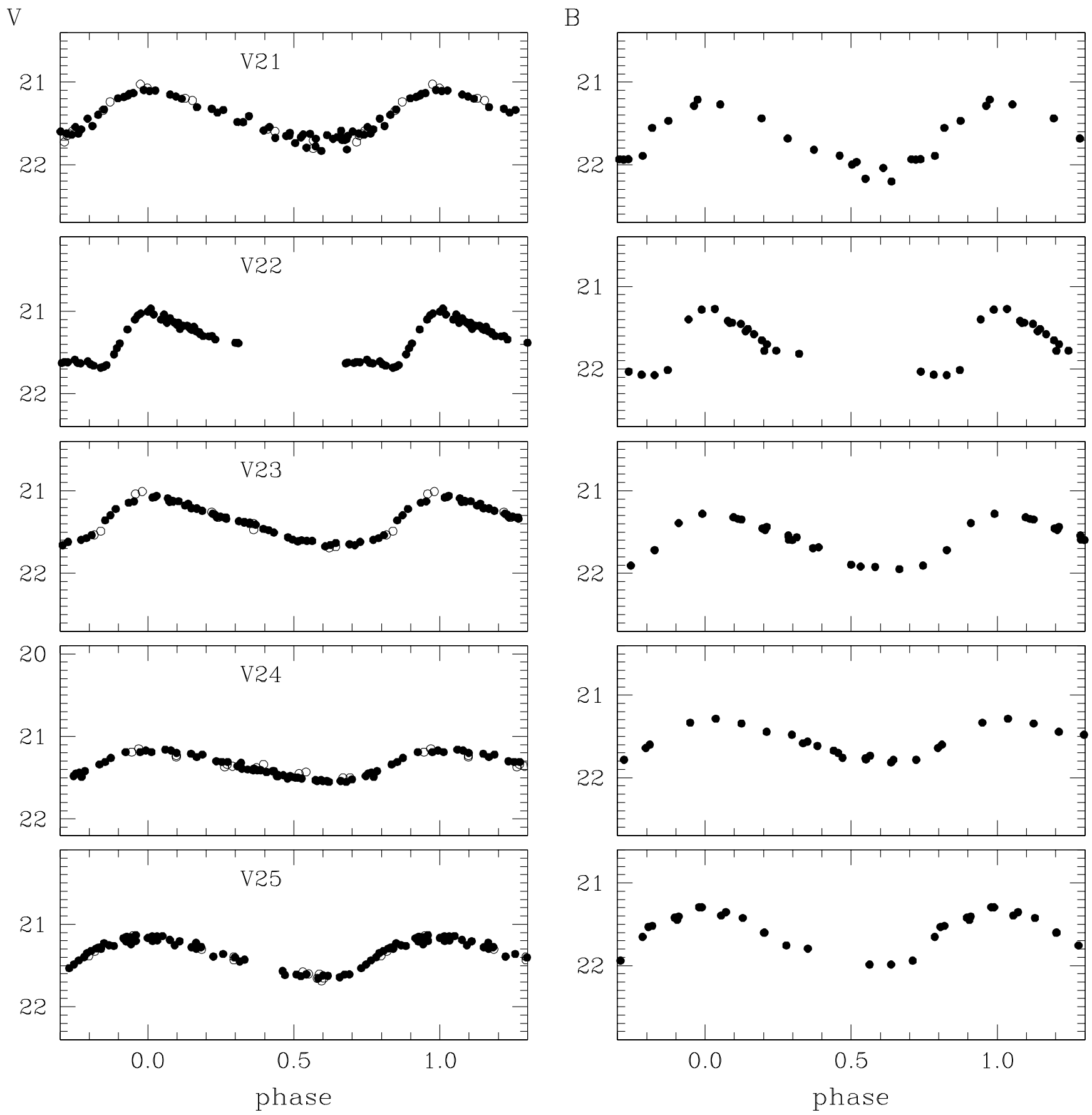

Fig. 4.- continued 
V
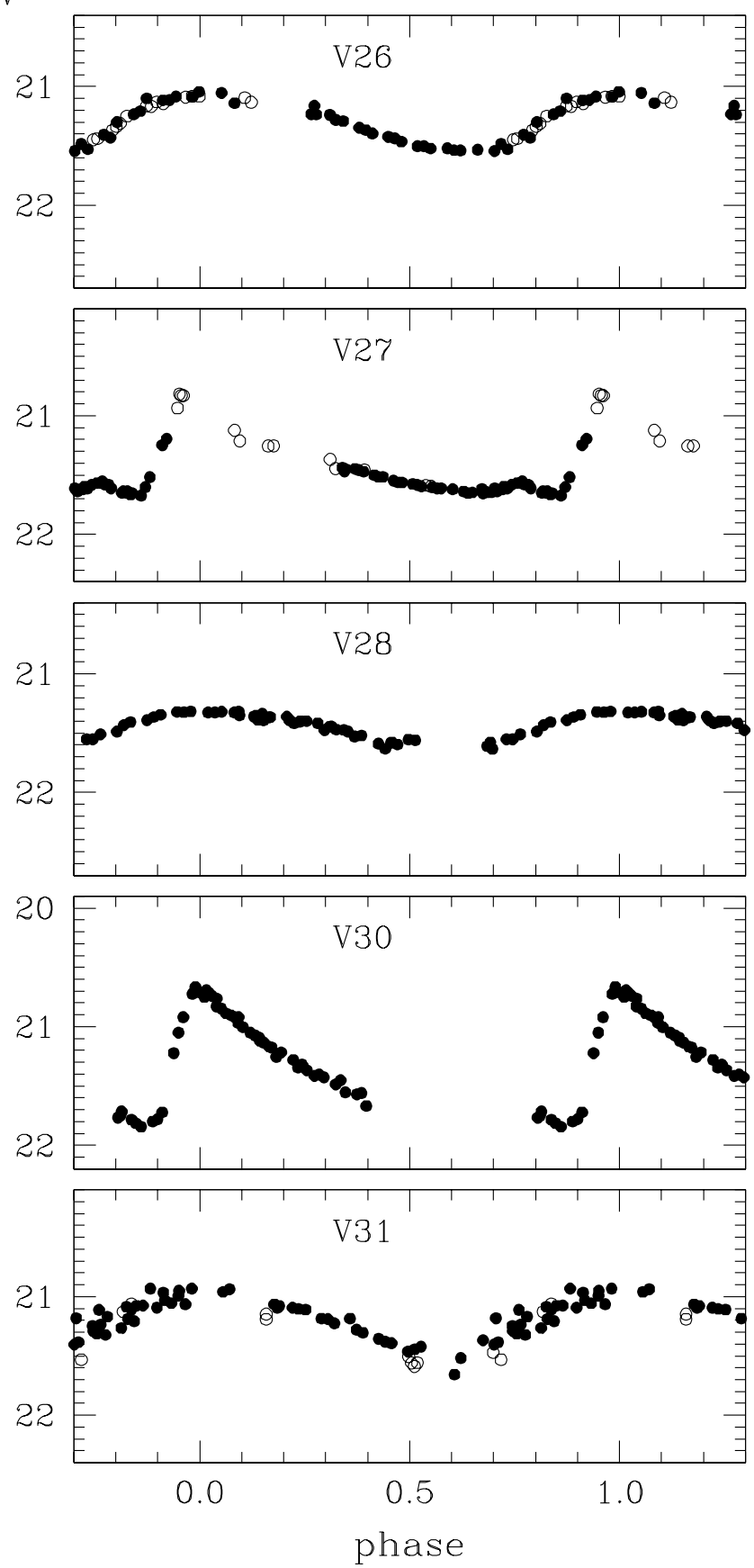

B
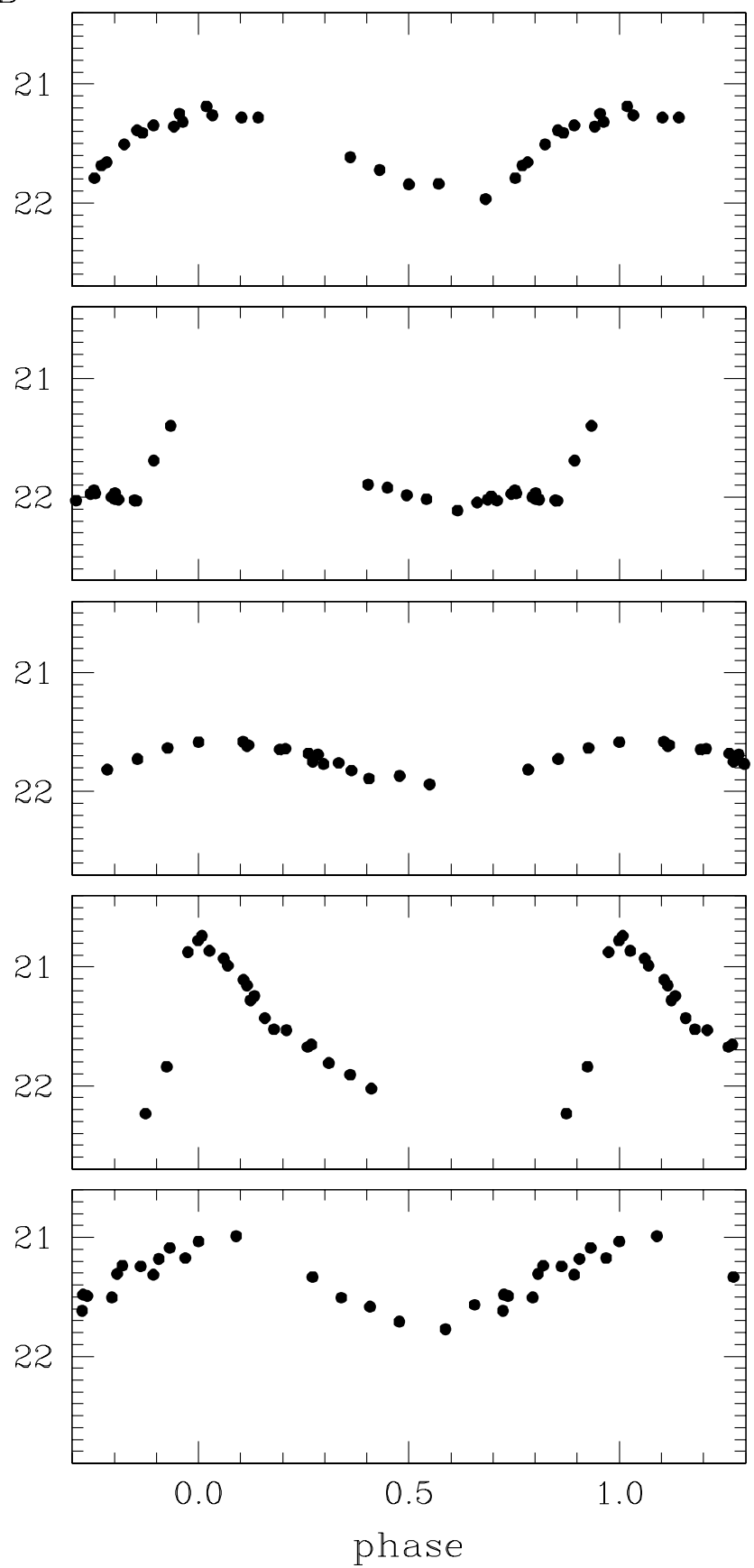

Fig. 4.- continued 


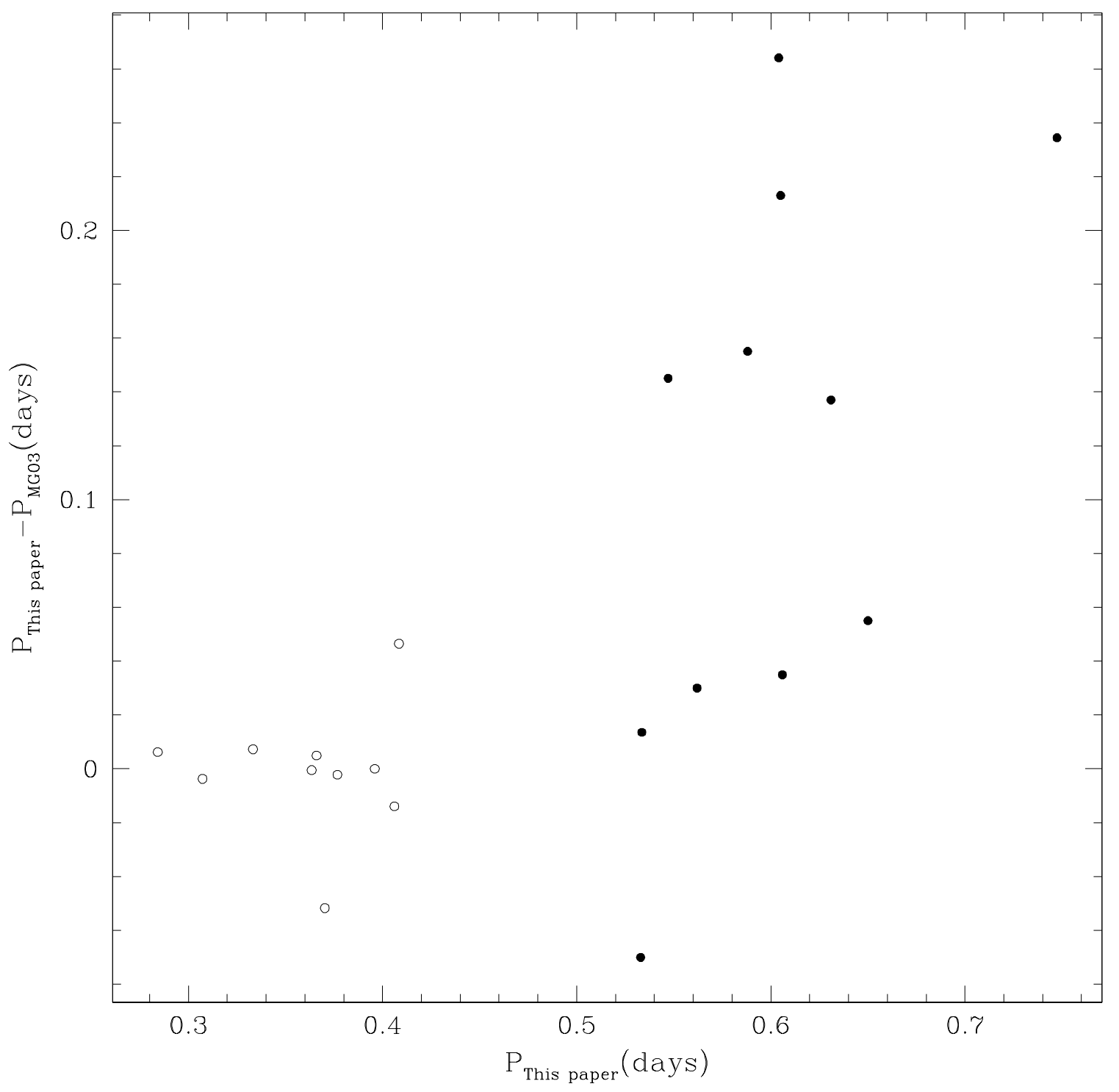

Fig. 5.- Comparison between our period determinations and MG03 values for the stars in common. Open and filled circles represent first-overtone and fundamental-mode pulsators, respectively, according to our type classification. 

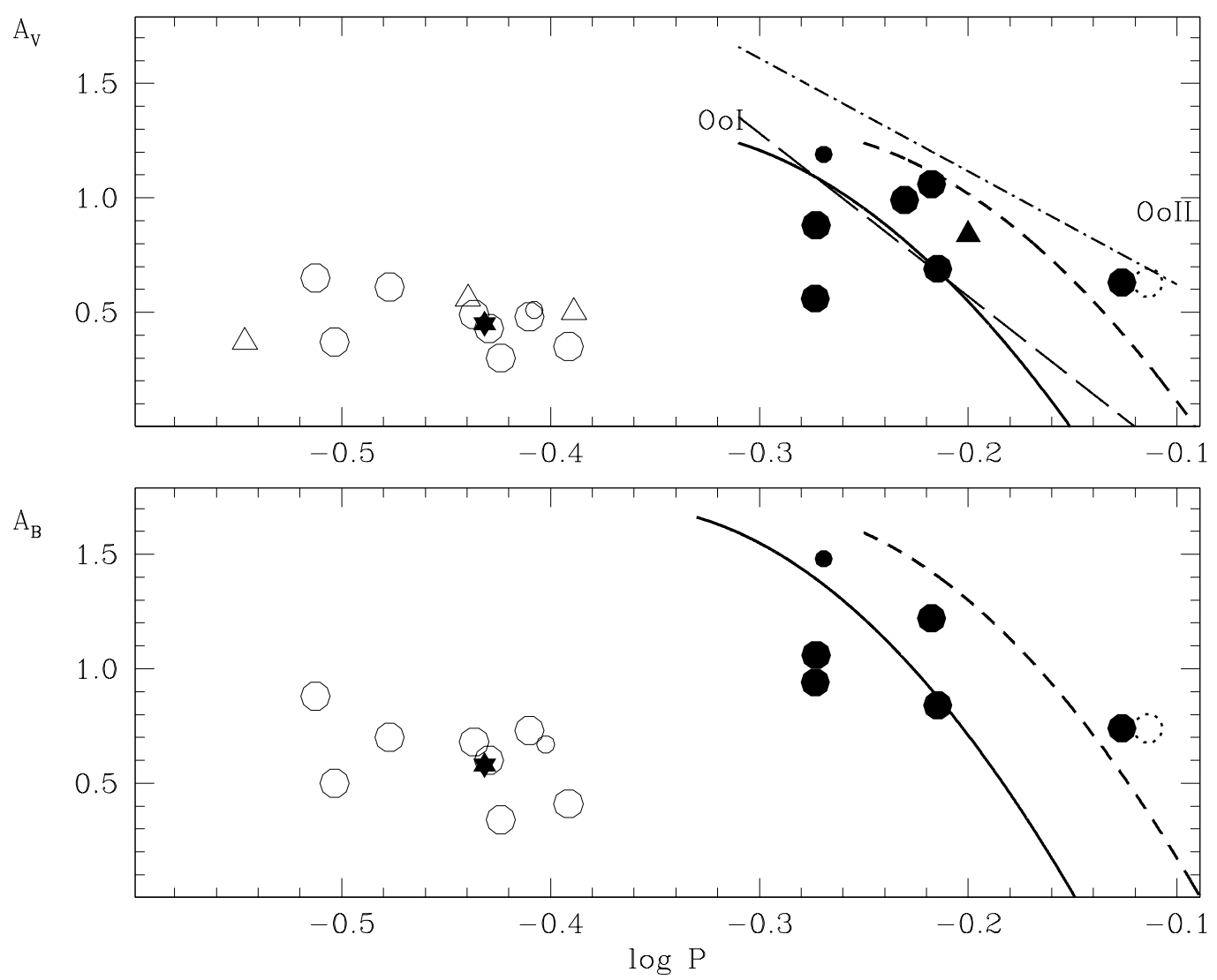

Fig. 6. - $V, B$ period-amplitude diagrams of the For 5 RR Lyrae stars. Filled circles: ab-type RR Lyrae stars; open circles: first-overtone RR Lyrae stars; star: candidate doublemode pulsator (RRd). Larger symbols indicate variable stars located at distances less $45^{\prime \prime}$ from the cluster center. Filled and open triangles are RRab and RRc variables within $8^{\prime \prime}$ from the center of For 5 which have light curves from the HST observations only. The dotted open circle shows the position of the RRab star V20 if the alternative longer period of 0.769393 days is adopted. The straight lines show the positions of the OoI and OoII Galactic GCs according to Clement \& Rowe (2000). Period-amplitude distributions of the bona fide regular (solid curves) and well-evolved (dashed curves) ab-type RR Lyrae stars in M3 from Cacciari, Corwin, \& Carney (2005) are also shown for comparison. 